\title{
THE OPTIMAL PROJECTION EQUATIONS FOR FINITE-DIMENSIONAL FIXED-ORDER DYNAMIC COMPENSATION OF INFINITE-DIMENSIONAL SYSTEMS*
}

\author{
DENNIS S. BERNSTEIN† AND DAVID C. HYLAND $\dagger$
}

\begin{abstract}
One of the major difficulties in designing implementable finite-dimensional controllers for distributed parameter systems is that such systems are inherently infinite dimensional while controller dimension is severely constrained by on-line computing capability. While some approaches to this problem initially seek a correspondingly infinite-dimensional control law whose finite-dimensional approximation may be of impractically high order, the usual engineering approach involves first approximating the distributed parameter system with a high-order discretized model followed by design of a relatively low-order dynamic controller. Among the numerous approaches suggested for the latter step are model/controller reduction techniques used in conjunction with the standard $L Q G$ result. An alternative approach, developed in [36], relies upon the discovery in [31] that the necessary conditions for optimal fixed-order dynamic compensation can be transformed into a set of equations possessing remarkable structural coherence. The present paper generalizes this result to apply directly to the distributed parameter system itself. In contrast to the pair of operator Riccati equations for the "full-order" $L Q G$ case, the optimal finite-dimensional fixed-order dynamic compensator is characterized by four operator equations (two modified Riccati equations and two modified Lyapunov equations) coupled by an oblique projection whose rank is precisely equal to the order of the compensator and which determines the optimal compensator gains. This "optimal projection" is obtained by a full-rank factorization of the product of the finite-rank nonnegative-definite Hilbert-space operators which satisfy the pair of modified Lyapunov equations. The coupling represents a graphic portrayal of the demise of the classical separation principle for the finite-dimensional reduced-order controller case. The results obtained apply to a semigroup formulation in Hilbert space and thus are applicable to control problems involving a broad range of specific partial and functional differential equations.
\end{abstract}

Key words. optimality conditions, finite-dimensional fixed-order dynamic compensator, infinitedimensional system, distributed parameter system, semisimple operator, oblique projection, Drazin generalized inverse

1. Introduction. One of the major difficulties in designing active controllers for distributed parameter systems is that such systems are inherently infinite dimensional while implementable controllers are necessarily finite dimensional with controller dimension severely constrained by on-line computing capability. As pointed out by Balas ([1], see also [2]), control design for distributed parameter systems entails the practical constraints of 1) finitely many sensors and actuators, 2) a finite-dimensional controller and 3) natural system dissipation. The validity of 2) is apparent from the fact that processing and transmitting electrical signals by conventional analog or digital components constitutes finite-dimensional action. Although distributed parameter devices can also be utilized, their fabrication and implementation can incorporate at most a finite number of design specifications. ${ }^{1}$ Hence, although distributed parameter systems are most accurately represented by infinite-dimensional models, real-world

* Received by the editors December 6, 1983, and in revised form September 15, 1984. This work was performed at Lincoln Laboratory/MIT and was sponsored by the Department of the Air Force.

$\dagger$ Harris Corporation, Government Aerospace Systems Division, Controls Analysis and Synthesis Group, Melbourne, Florida 32901.

${ }^{1}$ Examples of such components include tapped delay lines and surface acoustic wave devices. Although acoustoelectric convolvers [3, p. 465] can perform continuous-time integration, synthesis of the desired impulse-response kernel can incorporate only finitely many specified parameters. The obvious fact should also be noted that physical limitations impose an upper bound on the number of design parameters that can be incorporated in the construction of any device. For an extensive treatment of this subject, see [72]. 
constraints require that implementable controllers be modelled as lumped parameter systems.

Clearly, the above observations effectively preclude the possibility of realizing infinite-dimensional controllers that involve full-state feedback or full-state estimation (see, e.g., [4]-[6] and the numerous references therein). Although finite-dimensional approximation schemes have been applied to optimal infinite-dimensional control laws ([7]-[9]), these results only guarantee optimality in the limit, i.e., as the order of the approximating controller increases without bound. Hence, there is no guarantee that a particular approximate (i.e., discretized) controller is actually optimal over the class of approximate controllers of a given order dictated by implementation constraints. Moreover, even if an optimal approximate finite-dimensional controller could be obtained, it would almost certainly be suboptimal in the class of all controllers of the given order.

Although the usual engineering approach to this problem is to replace the distributed parameter system with a high-order finite-dimensional model, analogous, fundamental difficulties remain since application of $L Q G$ leads to a controller whose order is identical to that of the high-order approximate model. Attempts to remedy this problem usually rely upon some method of open-loop model reduction or closedloop controller reduction (see, e.g., [10]-[15]). Most of these techniques (with the exception of [11]) are ad hoc in nature, however, and hence guarantees of optimality and stability may be lacking.

A more direct approach that avoids both model and controller reduction is to fix the controller structure and optimize the performance criterion with respect to the controller parameters. Although much effort was devoted to this approach (see, e.g., [16]-[30]), progress in this direction was impeded by the extreme complexity of the nonlinear matrix equations arising from the first-order necessary conditions. What was lacking, to quote the insightful remarks of [24], was a "deeper understanding of the structural coherence of these equations." The key to unlocking these unwieldy equations was subsequently discovered by Hyland in [31] and developed in [32]-[36]. Specifically, it was found that these equations harbored the definition of an oblique projection (i.e., idempotent matrix) which is a consequence of optimality and not the result of an ad hoc assumption. By exploiting the presence of this "optimal projection," the originally very complex stationary conditions can be transformed without loss of generality into much simpler and more tractable forms. The resulting equations (see [36, (2.10)-(2.17)] ) preserve the simple form of $L Q G$ relations for the gains in terms of covariance and cost matrices which, in turn, are determined by a coupled system of two modified Riccati equations and two modified Lyapunov equations. This coupling, by means of the optimal projection, represents a graphic portrayal of the demise of the classical separation principle for the reduced-order controller case. When, as a special case, the order of the compensator is required to be equal to the order of the plant, the modified Riccati equations immediately reduce to the standard $L Q G$ Riccati equations and the modified Lyapunov equations express the proviso that the compensator be minimal, i.e., controllable and observable. Since the $L Q G$ Riccati equations as such are nothing more than the necessary conditions for full-order compensation, the "optimal projection equations" appear to provide a clear and simple generalization of standard $L Q G$ theory.

The fact that the optimal projection equations consist of four coupled matrix equations, i.e., two modified Riccati equations and two modified Lyapunov equations, can readily be explained by the following simple reason. Reduced-order control-design methods often involve either $L Q G$ applied to a reduced-order model or model reduction 
applied to a full-order $L Q G$ design, and hence both approaches require the solution of precisely four equations: two Riccati equations (for $L Q G$ ) plus two Lyapunov equations (for system reduction via balancing, as in [12], [14]). The coupled form of the optimal projection equations is thus a strong reminder that the $L Q G$ and orderreduction operations cannot be iterated but must, in a precise sense, be performed simultaneously. This situation is partly due to the fact that the optimal projection matrix may not be of the form [ $\left[\begin{array}{ll}I & 0 \\ 0\end{array}\right]$ even in the basis corresponding to the "balanced" realization [12], [14]. This point is explored in [37], [37a] where the solution to the optimal model-reduction problem is characterized by a pair of modified Lyapunov equations which are also coupled by an oblique projection.

Returning now to the distributed parameter problem, it should be mentioned that notable exceptions to the previously mentioned work on distributed parameter controllers are the contributions of Johnson [38] and Pearson [39], [40] who suggest fixing the order of the finite-dimensional compensator while retaining the distributed parameter model. Progress in this direction, however, was impeded not only by the intractability of the optimality conditions that were available for the finite-dimensional problem (as in [16]-[30]), but also by the lack of a suitable generalization of these conditions to the infinite-dimensional case. The purpose of the present paper is to make significant progress in filling these gaps, i.e., by deriving explicit optimality conditions which directly characterize the optimal finite-dimensional fixed-order dynamic compensator for an infinite-dimensional system and which are exactly analogous to the highly simplified optimal projection equations obtained in [31]-[34], [36] for the finite-dimensional case. Specifically, instead of a system for four matrix equations we obtain a system of four operator equations whose solutions characterize the optimal finite-dimensional fixed-order dynamic compensator. Moreover, the optimal projection now becomes a bounded idempotent Hilbert-space operator whose rank is precisely equal to the order of the compensator.

The mathematical setting we use is standard: a linear time-invariant differential system in Hilbert space with additive white noise, finitely many controls and finitely many noisy measurements (thus satisfying the first practical constraint mentioned above). The input and output maps are assumed to be bounded. Since the only explicit assumption on the unbounded dynamics operator is that it generate a strongly continuous semigroup, the results are potentially applicable to a broad range of specific partial and functional differential equations. The actual applicability of our results is essentially limited by practical constraint 3 ). Since we are concerned with the steadystate problem, we implicitly assume that the distributed parameter system is stabilizable, i.e., that there exists a dynamic compensator of a given order such that the closed-loop system is uniformly stable. We note that stabilizing compensators do exist for the wide class of problems considered in [41] and [42] which includes delay, parabolic and damped hyperbolic systems. The question of how much damping is required for stabilizability of hyperbolic systems is a crucial issue in designing controllers for large flexible space structures [7], [43]-[49a].

It is important to point out that the results of this paper can immediately be specialized to finite-dimensional systems by requiring that the Hilbert space characterizing the dynamical system be finite-dimensional. Then all unboundedness considerations can be ignored, adjoints can be interpreted as transposes and other obvious simplifications can be invoked. The only mathematical aspect requiring attention is the treatment of white noise which, for general handling of the infinite-dimensional case, is interpreted according to [6]. ${ }^{2}$ For the finite-dimensional case, however, the standard 
classical notions suffice and the results go through with virtually no modifications.

The contents of the paper are as follows. Section 2 contains preliminary notation in addition to particular results for use later in the paper. Section 3 presents the optimal steady-state finite-dimensional fixed-order dynamic-compensation problem and the Main Theorem gives the necessary conditions in the form of the optimal projection equations (3.15)-(3.18). We then develop a series of results which serve to elucidate several aspects of the Main Theorem. Section 4 is devoted to the proof of the Main Theorem. The reader is alerted to the two crucial steps required. The first step involves generalizing to the infinite-dimensional case the derivation of the necessary conditions in their "primitive" form (see (4.27)-(4.29) and (4.48)-(4.53)). The derivation in [31]-[33], [36] involving Lagrange multipliers is invalid in the infinite-dimensional case due to the presence of the unbounded system-dynamics operator. Instead, we use the gramian form of the closed-loop covariance operator to obtain a dual problem formulation and then proceed to derive the primitive necessary conditions by means of a lengthy, but direct, computation (Lemma 4.7). The second crucial step involves transforming the primitive form of the necessary conditions to the final form given in the Main Theorem. This laborious computation was first carried out in [31], [32] and was subsequently facilitated in [33], [36] by means of a judicious change of variables (see (4.32), (4.33)). Finally, some concluding remarks are given in $\S 5$.

2. Preliminaries. In this section we introduce general notation along with basic definitions and results for use in later sections. Our principal references are [6], [50] and [51].

Throughout this section let $\mathscr{H}, \mathscr{H}^{\prime}$ and $\mathscr{H}^{\prime \prime}$ denote real separable Hilbert spaces with norm $\|\cdot\|$ and inner product $\langle\cdot, \cdot\rangle$ and let $\mathscr{B}\left(\mathscr{H}, \mathscr{H}^{\prime}\right)$ denote the space of bounded linear operators from $\mathscr{H}$ into $\mathscr{H}^{\prime}$. For $L \in \mathscr{B}\left(\mathscr{H}, \mathscr{H}^{\prime}\right),\|L\|$ is the norm of $L, \mathscr{R}(L)$ is the range of $L, \mathcal{N}(L)$ is the null space of $L, \rho(L)$ is the rank of $L$ (set $\rho(L)=\infty$ if $L$ does not have finite rank), $L^{-1}$ is the inverse of $L$ when $L$ is invertible, i.e., when $L$ has a bounded inverse, $L^{*}$ is the adjoint of $L$ and $L^{-*} \triangleq\left(L^{*}\right)^{-1}$. Recall that $\|L\|=\left\|L^{*}\right\|$ and that $\rho(L)=\rho\left(L^{*}\right)\left[50\right.$, p. 161]. Now suppose that $\mathscr{H}=\mathscr{H}^{\prime}$ so that $L \in \mathscr{B}(\mathscr{H}) \triangleq \mathscr{B}(\mathscr{H}, \mathscr{H})$. If $L L^{*}=L^{*} L$ then $L$ is normal and if $L=L^{*}$ then $L$ is selfadjoint. If $L$ is selfadjoint and $\langle L x, x\rangle \geqq 0, x \in \mathscr{H}$, then $L$ is nonnegative definite. Note that the selfadjointness assumption is included in the definition since the Hilbert spaces are assumed real. If $L$ is nonnegative definite then $L^{1 / 2}$ denotes the (unique) nonnegative-definite square root of $L$. Call $L$ semisimple (resp., real semisimple, nonnegative semisimple) if there exists invertible $S \in \mathscr{B}(\mathscr{H})$ such that $S L S^{-1}$ is normal (resp., selfadjoint, nonnegative definite). This implies that $S L S^{-1}$ has a complete set of orthonormal eigenvectors and, in the real-semisimple or nonnegative-semisimple cases, has real or nonnegative eigenvalues.

Recall that if $L \in \mathscr{B}(\mathscr{H})$ is compact then $L$ has at most a countable number of eigenvalues and all nonzero eigenvalues have finite multiplicity. Hence, for $L \in$ $\mathscr{B}\left(\mathscr{H}, \mathscr{H}^{\prime}\right)$ compact, let $\left\{\alpha_{i}\right\}$ be the (at most countable) sequence of eigenvalues of $\left(L L^{*}\right)^{1 / 2}$ with appropriate multiplicity and $\alpha_{1} \geqq \alpha_{2} \geqq \cdots>0[50$, p. 261]. Then $\mathscr{B}_{1}\left(\mathscr{H}, \mathscr{C}^{\prime}\right)$ denotes the set of trace class (or nuclear) operators, i.e., the set of compact

\footnotetext{
${ }^{2}$ Alternatively, we could have adopted the white noise formulation of [4]. The main difference between the two white noise formalisms is that Balakrishnan works with finitely additive rather than countably additive measures. Strictly speaking, then, even in finite dimensions Balakrishnan's white noise is different from the standard notion (see $[6, \mathrm{pp} .307,315])$.
} 
$L \in \mathscr{B}\left(\mathscr{H}, \mathscr{H}^{\prime}\right)$ for which $\sum_{i} \alpha_{i}<\infty\left[50\right.$, p. 521]. $\mathscr{B}_{1}\left(\mathscr{H}, \mathscr{H}^{\prime}\right)$ is a Banach space with norm

$$
\|L\|_{1} \triangleq \sum_{i} \alpha_{i}
$$

If $\sum_{i} \alpha_{i}^{2}<\infty$ then $L \in \mathscr{B}_{2}\left(\mathscr{H}, \mathscr{C}^{\prime}\right)$, the set of Hilbert-Schmidt operators, which is a Banach space with norm

$$
\|L\|_{2} \triangleq\left[\sum_{i} \alpha_{i}^{2}\right]^{1 / 2}
$$

Note that $\|L\| \leqq\|L\|_{2} \leqq\|L\|_{1},\|L\|=\left\|L^{*}\right\|,\|L\|_{1}=\left\|L^{*}\right\|_{1}$ and $\|L\|_{2}=\left\|L^{*}\right\|_{2}$. If $\mathscr{H}=\mathscr{H}^{\prime}$, then we write $\mathscr{B}_{1}(\mathscr{H})$ and $\mathscr{B}_{2}(\mathscr{H})$ for $\mathscr{B}_{1}(\mathscr{H}, \mathscr{H})$ and $\mathscr{B}_{2}(\mathscr{H}, \mathscr{H})$, respectively. Note that if nonnegative-definite $L \in \mathscr{B}_{1}(\mathscr{H})$ then $L^{1 / 2} \in \mathscr{B}_{2}(\mathscr{H})$.

If $L \in \mathscr{B}_{1}\left(\mathscr{H}, \mathscr{H}^{\prime}\right)$ and $S \in \mathscr{B}\left(\mathscr{H}^{\prime}, \mathscr{H}^{\prime \prime}\right)$ then

$$
\|S L\|_{1} \leqq\|S\|\|L\|_{1}
$$

and hence $S L \in \mathscr{B}_{1}\left(\mathscr{H}, \mathscr{H}^{\prime}\right)$. Similarly, under suitable hypotheses,

$$
\|L S\|_{1} \leqq\|S\|\|L\|_{1},
$$

and

$$
\|S L\|_{1} \leqq\|S\|_{2}\|L\|_{2} .
$$

Lemma 2.1. Suppose $L \in \mathscr{B}_{1}(\mathscr{H})$ and let $\left\{\lambda_{i}\right\}$ denote the nonzero eigenvalues of $L$ with appropriate multiplicity. Then $[51, p .89]$

$$
\sum_{i}\left|\lambda_{i}\right| \leqq\|L\|_{1}
$$

If $L$ is selfadjoint then $[50, p .522]$

$$
\sum_{i}\left|\lambda_{i}\right|=\|L\|_{1}
$$

If $L$ is nonnegative definite then

$$
\sum_{i} \lambda_{i}=\|L\|_{1}
$$

Let $L \in \mathscr{B}_{1}(\mathscr{H})$. Then define $\left[50\right.$, p. 523] the trace functional $\operatorname{tr}: \mathscr{B}_{1}(\mathscr{H}) \rightarrow \mathbb{R}$ by

$$
\operatorname{tr} L \triangleq \sum_{i}\left\langle L \phi_{i}, \phi_{i}\right\rangle,
$$

where the summation is independent of the choice of orthonormal basis $\left\{\phi_{i}\right\}$. The trace satisfies $\operatorname{tr} L=\operatorname{tr} L^{*}, \operatorname{tr} S L=\operatorname{tr} L S$ for all $S \in \mathscr{B}(\mathscr{H}), \operatorname{tr} S T=\operatorname{tr} T S$ for all $S, T \in \mathscr{B}_{2}(\mathscr{H})$ and $\operatorname{tr}(\alpha T+\beta S)=\alpha(\operatorname{tr} T)+\beta(\operatorname{tr} S)$ for all $\alpha, \beta \in \mathbb{R}$ and $S, T \in \mathscr{B}_{1}(\mathscr{H})$.

Lemma 2.2. Suppose $L \in \mathscr{B}_{1}(\mathscr{H})$ and let $\left\{\lambda_{i}\right\}$ denote the nonzero eigenvalues of $L$ with appropriate multiplicity. Then $[51, p .139]$

$$
\operatorname{tr} L=\sum_{i} \lambda_{i}
$$

and hence (by Lemma 2.1)

$$
|\operatorname{tr} L| \leqq\|L\|_{1}
$$

If $L$ is nonnegative definite then

$$
\operatorname{tr} L=\|L\|_{1} .
$$


Corollary 2.1. For each $S \in \mathscr{B}(\mathscr{H})$ the linear functionals

$$
\begin{aligned}
& L \rightarrow \operatorname{tr} S L: \mathscr{B}_{1}(\mathscr{H}) \rightarrow \mathbb{R}, \\
& L \rightarrow \operatorname{tr} L S: \mathscr{B}_{1}(\mathscr{H}) \rightarrow \mathbb{R}
\end{aligned}
$$

are continuous. For each $L \in \mathscr{B}_{1}(\mathscr{H})$ the linear functionals

are continuous.

$$
\begin{aligned}
& S \rightarrow \operatorname{tr} L S: \mathscr{B}(\mathscr{H}) \rightarrow \mathbb{R}, \\
& S \rightarrow \operatorname{tr} S L: \mathscr{B}(\mathscr{H}) \rightarrow \mathbb{R}
\end{aligned}
$$

Although showing that a bounded linear operator is trace class is slightly more involved than the above characterizations of $\mathscr{B}_{1}(\mathscr{H})$, the following result will suffice for our purposes (see [52, p. 96], or [52a, p. 171]).

LeMMA 2.3. Let $L \in \mathscr{B}(\mathscr{H})$ be nonnegative definite. Then

$$
\sum_{i}\left\langle L \phi_{i}, \phi_{i}\right\rangle
$$

whether finite or infinite, is independent of the orthonormal basis $\left\{\phi_{i}\right\}$. The summation is finite if and only if $L \in \mathscr{B}_{1}(\mathscr{H})$.

Many of the operators introduced in the following section have finite-dimensional domain or range space and hence are degenerate, i.e., have finite rank. Recall that degenerate operators are necessarily trace class. The following result, which generalizes $[53, T h m .2 .1$, p. 240$]$ in certain respects, will be fundamental in decomposing finite-rank operators.

Lemma 2.4. Suppose $L_{1}, \cdots, L_{r} \in B\left(\mathscr{H}, \mathscr{H}^{\prime}\right)$ have finite rank. Then there exists a finite-dimensional subspace $\mathcal{M} \subset \mathscr{H}$ such that $L_{i} \mathcal{M}^{\perp}=0, i=1, \cdots, r$. Furthermore, if $\mathscr{H}=\mathscr{H}^{\prime}$ then $\mathcal{M}$ can be chosen such that $L_{i} \mathcal{M} \subset \mathcal{M}, i=1, \cdots, r$.

Proof. It suffices to consider the case $r=1$. Writing $L$ for $L_{1}$, note that since $\rho\left(L^{*}\right)<\infty, \mathcal{N}(L)^{\perp}=\mathscr{R}\left(L^{*}\right)[50$, p. 155] and $\mathcal{N}(L)$ is closed, the first statement holds with $\mathcal{M}=\mathcal{N}(L)^{\perp}$. When $\mathscr{H}=\mathscr{H}^{\prime}$ set $\mathcal{M}=\mathcal{N}(L)^{\perp}+\mathscr{R}(L)$ and note that $M^{\perp}=$ $\mathcal{N}(L) \cap \mathscr{R}(L)^{\perp} \subset \mathcal{N}(L)$ and $L \mathcal{M} \subset \mathscr{R}(L) \subset \mathcal{M}$.

The following generalization of Sylvester's inequality [54, p. 66] will be used repeatedly in handling finite-rank operators.

Lemma 2.5. Let $L \in \mathscr{B}\left(\mathscr{H}, \mathscr{H}^{\prime}\right)$ and $S \in \mathscr{B}\left(\mathscr{H}^{\prime}, \mathscr{H}^{\prime \prime}\right)$. Then

$$
\rho(S L) \leqq \min \{\rho(S), \rho(L)\} .
$$

If $\operatorname{dim} \mathscr{H}^{\prime}=\nu<\infty$, then

$$
\rho(S)+\rho(L)-\nu \leqq \rho(S L) .
$$

Proof. If either $S$ or $L$ does not have finite rank then (2.1) is immediate. If both $S$ and $L$ have finite rank then the standard arguments [54] used to prove the finitedimensional version of (2.1) remain valid. To prove (2.2), note that Lemma 2.4 implies that there exist orthonormal bases for $\mathscr{H}$ and $\mathscr{H}^{\prime}$ with respect to which $L$ has the matrix representation $\left[\begin{array}{cl}\tilde{L} & 0\end{array}\right]$, where $\tilde{L} \in \mathbb{R}^{\nu \times p}$. Similarly, there exist orthonormal bases for $\mathscr{H}^{\prime}$ and $\mathscr{H}^{\prime \prime}$ with respect to which $S$ has the matrix representation $\left[\begin{array}{c}\tilde{S} \\ 0\end{array}\right]$, where $\tilde{S} \in \mathbb{R}^{q \times \nu}$. Since the two cited bases for $\mathscr{H}^{\prime}$ may be different, let orthogonal $U \in \mathbb{R}^{\nu \times \nu}$ be the matrix representation (with respect to either basis for $\mathscr{H}^{\prime}$ ) for the change in orthonormal basis $\left[6\right.$, p. 100]. Hence $S L$ has the matrix representation $\left[\begin{array}{cc}\tilde{S} U \tilde{L} & 0 \\ 0 & 0\end{array}\right]$ and $(2.2)$ follows from the known result $[54$, p. 66$]$.

As in the proof of Lemma 2.5, we shall utilize the infinite-matrix representation of an operator with respect to an orthonormal basis. All matrix representations given 
here will consist of real entries since the Hilbert spaces involved are real. When the orthonormal bases are specified and no confusion can arise, we shall not differentiate between an operator and its matrix representation. We shall use the infinite identity matrix $I_{\infty}$ interchangeably with the identity $I_{\mathscr{H}}$ on $\mathscr{H}$.

When dealing with finite-dimensional Euclidean spaces the notation and terminology introduced above will be utilized with only minor changes. For example, bounded linear operators will be represented by matrices whose elements are determined according to fixed orthonormal bases and hence we identify $\mathbb{R}^{m \times n}=\mathscr{B}\left(\mathbb{R}^{n}, \mathbb{R}^{m}\right)$. Note that if $L \in \mathscr{B}\left(\mathbb{R}^{n}, \mathscr{H}\right)$ and $S \in \mathscr{B}\left(\mathscr{H}, \mathbb{R}^{m}\right)$ then $S L$ is an $m \times n$ matrix which is independent of any particular orthonormal basis for $\mathscr{H}$. The transposes of $x \in \mathbb{R}^{n} \triangleq \mathbb{R}^{n \times 1}$ and $M \in \mathbb{R}^{m \times n}$ are denoted by $x^{T}$ and $M^{T}$ and $M^{-T} \triangleq\left(M^{T}\right)^{-1}$. Let $I_{n}$ denote the $n \times n$ identity matrix.

To specialize some of the above operator terminology to matrices, let $M \in \mathbb{R}^{n \times n}$. We shall say $M$ is nonnegative (resp., positive) diagonal if $M$ is diagonal with nonnegative (resp., positive) diagonal elements. $M$ is nonnegative (resp., positive) definite if $M$ is symmetric and $x^{T} M x \geqq 0$ (resp., $x^{T} M x>0$ ), $x \in \mathbb{R}^{n}$. Recall that $M$ is symmetric (resp., nonnegative definite, positive definite) if and only if there exists orthogonal $U \in \mathbb{R}^{n \times n}$ such that $U M U^{T}$ is diagonal (resp., nonnegative diagonal, positive diagonal). $M$ is semisimple [55, p. 13], or nondefective [56, p. 375], if $M$ has $n$ linearly independent eigenvectors, i.e., $M$ has a diagonal Jordan canonical form over the complex field. $M$ is real (resp., nonnegative, positive) semisimple if $M$ is semisimple with real (resp., nonnegative, positive) eigenvalues. Note that $M$ is real (resp., nonnegative, positive) semisimple if and only if there exists invertible $S \in \mathbb{R}^{n \times n}$ such that $S M S^{-1}$ is diagonal (resp., nonnegative diagonal, positive diagonal). Alternatively, $M$ is real (resp., nonnegative, positive) semisimple if and only if there exists invertible $S \in \mathbb{R}^{n \times n}$ such that $S M S^{-1}$ is symmetric (resp., nonnegative definite, positive definite).

LEMMA 2.6. The product of two nonnegative- (resp., positive-) definite matrices is nonnegative (resp., positive) semisimple.

Proof. If $S, L \in \mathbb{R}^{n \times n}$ are both nonnegative (resp., positive) definite then by [55, Thm. 6.2.5, p. 123] there exists invertible $\phi \in \mathbb{R}^{n \times n}$ such that $D_{S} \triangleq \phi^{-1} S \phi^{-T}$ and $D_{L} \triangleq \phi^{T} L \phi$ are nonnegative (resp., positive) diagonal. Hence, $S L=\phi D_{S} D_{L} \phi^{-1}$ is nonnegative (resp., positive) semisimple, as desired. Alternatively, if either $S$ or $L$ is positive definite, then the result follows from $S L=L^{-1 / 2}\left(L^{1 / 2} S L^{1 / 2}\right) L^{1 / 2}$ if $L$ is positive definite or $S L=S^{1 / 2}\left(S^{1 / 2} L S^{1 / 2}\right) S^{-1 / 2}$ if $S$ is positive definite.

3. Problem statement and the Main Theorem. We consider the following steadystate fixed-order dynamic-compensation problem. Given the dynamical system on $[0, \infty)$

$$
\begin{aligned}
& \dot{x}(t)=A x(t)+B u(t)+H_{1} w(t), \\
& y(t)=C x(t)+H_{2} w(t),
\end{aligned}
$$

design a finite-dimensional fixed-order dynamic compensator

$$
\begin{aligned}
& \dot{x}_{c}(t)=A_{c} x_{c}(t)+B_{c} y(t), \\
& u(t)=C_{c} x_{c}(t)
\end{aligned}
$$

which minimizes the steady-state performance criterion

$$
J\left(A_{c}, B_{c}, C_{c}\right) \triangleq \lim _{t \rightarrow \infty} \mathbb{E}\left[\left\langle R_{1} x(t), x(t)\right\rangle+u(t)^{T} R_{2} u(t)\right] .
$$


The following data are assumed. The state $x(t)$ is an element of a real separable Hilbert space $\mathscr{H}$ and the state differential equation is interpreted in the weak sense (see, e.g., [6, pp. 229, 317]). The closed, densely defined operator $A: \mathscr{D}(A) \subset \mathscr{H} \rightarrow \mathscr{H}$ generates a strongly continuous semigroup $e^{A t}, t \geqq 0$. The control $u(t) \in \mathbb{R}^{m}, B \in$ $\mathscr{B}\left(\mathbb{R}^{m}, \mathscr{H}\right)$ and the operator $R_{1} \in \mathscr{B}_{1}(\mathscr{H})$ and the matrix $R_{2} \in \mathbb{R}^{m \times m}$ are nonnegative definite and positive definite, respectively. $w(\cdot)$ is a zero-mean Gaussian "standard white noise process" in $L_{2}\left((0, \infty), \mathscr{H}^{\prime}\right)$ (see [6, p. 314]), where $\mathscr{H}^{\prime}$ is a real separable Hilbert space, $H_{1} \in \mathscr{B}_{2}\left(\mathscr{H}^{\prime}, \mathscr{H}\right), H_{2} \in \mathscr{B}\left(\mathscr{H}^{\prime}, \mathbb{R}^{l}\right)$ and " $E$ " denotes expectation. We assume that $H_{1} H_{2}^{*}=0$, i.e., the disturbance and measurement noises are independent, ${ }^{3}$ and that $V_{2} \triangleq H_{2} H_{2}^{*} \in \mathbb{R}^{l}$ is positive definite, i.e., all measurements are noisy. Note that $V_{1} \triangleq H_{1} H_{1}^{*} \in \mathscr{B}_{1}(\mathscr{H})$ is nonnegative definite and trace class. ${ }^{4}$ The initial state $x(0)$ is Gaussian and independent of $w(\cdot)$. The observation $y(t) \in \mathbb{R}^{l}$ and $C \in \mathscr{B}\left(\mathscr{H}, \mathbb{R}^{l}\right)$. The dimension of the compensator state $x_{c}(t)$ is of fixed, finite order $n_{c} \leqq \operatorname{dim} \mathscr{H}$ and the optimization is performed over $A_{c} \in \mathbb{R}^{n_{c} \times n_{c}}, B_{c} \in \mathbb{R}^{n_{c} \times l}$ and $C_{c} \in \mathbb{R}^{m \times n_{c}}$.

To handle the closed-loop system (3.1)-(3.4), we introduce the augmented state space $\tilde{\mathscr{H}} \triangleq \mathscr{H} \oplus \mathbb{R}^{n_{c}}$ which is a real separable Hilbert space with inner product $\left\langle\tilde{x}_{1}, \tilde{x}_{2}\right\rangle \triangleq$ $\left\langle x_{1}, x_{2}\right\rangle+x_{c 1}^{T} x_{c 2}, \tilde{x}_{i} \triangleq\left(x_{i}, x_{c i}\right)$. An operator $L \in \mathscr{B}(\tilde{\mathscr{C}})$ has a "decomposition" into operators $L_{1} \in \mathscr{B}(\mathscr{H}), L_{12} \in \mathscr{B}\left(\mathbb{R}^{n_{c}}, \mathscr{H}\right), L_{21} \in \mathscr{B}\left(\mathscr{H}, \mathbb{R}^{n_{c}}\right)$ and $L_{2} \in \mathbb{R}^{n_{c} \times n_{c}}$ in the sense that for $\tilde{x} \triangleq\left(x, x_{c}\right) \in \tilde{\mathscr{H}}, L \tilde{x}=\left(L_{1} x+L_{12} x_{c}, L_{21} x+L_{2} x_{c}\right)$, or, in "block" form,

$$
L=\left[\begin{array}{ll}
L_{1} & L_{12} \\
L_{21} & L_{2}
\end{array}\right] \text {. }
$$

For later use note that

$$
\|L\| \leqq\left\|L_{1}\right\|+\left\|L_{12}\right\|+\left\|L_{21}\right\|+\left\|L_{22}\right\|
$$

and

$$
L^{*}=\left[\begin{array}{ll}
L_{1}^{*} & L_{21}^{*} \\
L_{12}^{*} & L_{2}^{T}
\end{array}\right]
$$

We can similarly construct unbounded operators in $\tilde{\mathscr{H}}$. Hence, define the closedloop dynamics operator $\tilde{A}: \mathscr{D}(\tilde{A}) \subset \tilde{\mathscr{H}} \rightarrow \tilde{\mathscr{H}}$ on the dense domain $\mathscr{D}(\tilde{A}) \triangleq \mathscr{D}(A) \times \mathbb{R}^{n_{c}}$ by $\tilde{A} \tilde{x}=\left(A x+B C_{c} x_{c}, B_{c} C x+A_{c} x_{c}\right)$. Since $\tilde{A}$ can be represented by

$$
\tilde{A}=\left[\begin{array}{cc}
A & B C_{c} \\
B_{c} C & A_{c}
\end{array}\right]=\left[\begin{array}{cc}
A & 0 \\
0 & 0
\end{array}\right]+\left[\begin{array}{cc}
0 & B C_{c} \\
B_{c} C & A_{c}
\end{array}\right]
$$

and since the closed-loop operator

$$
\left[\begin{array}{ll}
A & 0 \\
0 & 0
\end{array}\right]: \mathscr{D}(\tilde{A}) \rightarrow \tilde{\mathscr{H}}
$$

generates the strongly continuous semigroup

$$
\left[\begin{array}{cc}
e^{A t} & 0 \\
0 & I_{n_{c}}
\end{array}\right], \quad t \geqq 0,
$$

it follows from [50, Thm., p. 497] that $\tilde{A}$ is also closed and generates a strongly continuous semigroup $e^{\tilde{A} t} \in \mathscr{B}(\tilde{\mathscr{H}}), t \geqq 0$. To guarantee that $J$ is finite and independent

\footnotetext{
${ }^{3}$ This assumption and its analogue, the lack of a cross-weighting term $x(t)^{T} R_{12} u(t)$ in (3.5), are for convenience only. See $\$ 5$.

${ }^{4}$ We must require that $R_{1}$ and $V_{1}$ be nuclear since covariance operators in the white noise formulation of [6] are not necessarily trace class as they are in the formulation of [4].
} 
of initial conditions we restrict our attention to the set of admissible stabilizing compensators

$$
\mathscr{A} \triangleq\left\{\left(A_{c}, B_{c}, C_{c}\right): e^{\tilde{A} t} \text { is exponentially stable }\right\} .
$$

Hence if $\left(A_{c}, B_{c}, C_{c}\right) \in \mathscr{A}$ then there exist $\alpha>0$ and $\beta>0$ such that

$$
\left\|e^{\tilde{A} t}\right\| \leqq \alpha e^{-\beta t}, \quad t \geqq 0 .
$$

Since the value of $J$ is independent of the internal realization of the compensator, we can further restrict our attention to

$$
\mathscr{A}_{+} \triangleq\left\{\left(A_{c}, B_{c}, C_{c}\right) \in \mathscr{A}:\left(A_{c}, B_{c}\right) \text { is controllable and }\left(C_{c}, A_{c}\right) \text { is observable }\right\} \text {. }
$$

The following lemma is required for the statement of the Main Theorem.

LEMMA 3.1. Suppose $\hat{Q}, \hat{P} \in \mathscr{B}(\mathscr{H})$ have finite rank and are nonnegative definite. Then $\hat{Q} \hat{P}$ is nonnegative semisimple. Furthermore, if $\rho(\hat{Q} \hat{P})=n_{c}$ then there exist $G$, $\Gamma \in \mathscr{B}\left(\mathscr{H}, \mathbb{R}^{n_{c}}\right)$ and positive-semisimple $M \in \mathbb{R}^{n_{c} \times n_{c}}$ such that

$$
\begin{aligned}
& \hat{Q} \hat{P}=G^{*} M \Gamma, \\
& \Gamma G^{*}=I_{n_{c^{*}}}
\end{aligned}
$$

Proof. By Lemma 2.4 there exists a finite-dimensional subspace $M \subset \mathscr{H}$ such that $\hat{Q} M \subset \mathcal{Q}, \hat{Q} M^{\perp}=0, \hat{P} M \subset \mathcal{M}$ and $\hat{P} M^{\perp}=0$. Hence there exists an orthonormal basis for $\mathscr{H}$ with respect to which $\hat{Q}$ and $\hat{P}$ have the infinite-matrix representations

$$
\hat{Q}=\left[\begin{array}{cc}
\hat{Q}_{1} & 0 \\
0 & 0
\end{array}\right], \quad \hat{P}=\left[\begin{array}{cc}
\hat{P}_{1} & 0 \\
0 & 0
\end{array}\right]
$$

where $\hat{Q}_{1}, \hat{P}_{1} \in \mathbb{R}^{r \times r}$ are nonnegative definite and $r \triangleq \operatorname{dim} \mu$. Since by Lemma 2.6 there exists invertible $\Psi \in \mathbb{R}^{r \times r}$ such that $\tilde{\Lambda}=\Psi^{-1} \hat{Q}_{1} \hat{P}_{1} \Psi$ is nonnegative diagonal, we have

$$
\hat{Q} \hat{P}=\left[\begin{array}{cc}
\Psi & 0 \\
0 & I_{\infty}
\end{array}\right]\left[\begin{array}{cc}
\tilde{\Lambda} & 0 \\
0 & 0
\end{array}\right]\left[\begin{array}{cc}
\Psi^{-1} & 0 \\
0 & I_{\infty}
\end{array}\right],
$$

which shows that $\hat{Q} \hat{P}$ is nonnegative semisimple. If, furthermore, $\rho(\hat{Q} \hat{P})=n_{c}$ then it is clear that $\Psi$ can be chosen (i.e., modified by an orthogonal matrix) so that

$$
\tilde{\Lambda}=\left[\begin{array}{ll}
\Lambda & 0 \\
0 & 0
\end{array}\right] \text {, }
$$

where $\Lambda \in \mathbb{R}^{n_{\mathrm{c}} \times n_{\mathrm{c}}}$ is positive diagonal. Hence,

$$
\left.\hat{Q} \hat{P}=\left[\begin{array}{cc}
\Psi & 0 \\
0 & I_{\infty}
\end{array}\right]\left[\begin{array}{c}
I_{n_{c}} \\
0
\end{array}\right]\right] \Lambda\left[\left[\begin{array}{ll}
I_{n_{c}} & 0
\end{array}\right] \quad 0\right]\left[\begin{array}{cc}
\Psi^{-1} & 0 \\
0 & I_{\infty}
\end{array}\right]
$$

which shows that (3.7) and (3.8) are satisfied with

$$
\left.G=\left[\begin{array}{ll}
S^{T} & 0
\end{array}\right][0]\left[\begin{array}{cc}
\Psi^{T} & 0 \\
0 & I_{\infty}
\end{array}\right], \quad M=S^{-1} \Lambda S, \Gamma=\left[\begin{array}{ll}
S^{-1} & 0
\end{array}\right] \quad 0\right]\left[\begin{array}{cc}
\Psi^{-1} & 0 \\
0 & I_{\infty}
\end{array}\right],
$$

for all invertible $S \in \mathbb{R}^{n_{c} \times n_{c}}$.

We shall refer to $G, \Gamma \in \mathscr{B}\left(\mathscr{H}, \mathbb{R}^{n_{c}}\right)$ and positive-semisimple $M \in \mathbb{R}^{n_{c} \times n_{c}}$ satisfying (3.7) and (3.8) as a $(G, M, \Gamma)$-factorization of $\hat{Q} \hat{P}$. For convenience in stating the Main Theorem define

$$
\sum \triangleq B R_{2}^{-1} B^{*}, \quad \bar{\Sigma} \triangleq C^{*} V_{2}^{-1} C
$$


Main Theorem. Suppose $\left(A_{c}, B_{c}, C_{c}\right) \in \mathscr{A}_{+}$solves the steady-state fixed-order dynamic-compensation problem. Then there exist nonnegative-definite $Q, P, \hat{Q}, \hat{P} \in \mathscr{B}_{1}(\mathscr{H})$ such that $A_{c}, B_{c}$ and $C_{c}$ are given by

$$
\begin{aligned}
& A_{c}=\Gamma(A-Q \bar{\Sigma}-\Sigma P) G^{*}, \\
& B_{c}=\Gamma Q C^{*} V_{2}^{-1}, \\
& C_{c}=-R_{2}^{-1} B^{*} P G^{*},
\end{aligned}
$$

for some $(G, M, \Gamma)$-factorization of $\hat{Q} \hat{P}$, and such that, with $\tau \triangleq G^{*} \Gamma$, the following conditions are satisfied:

$$
Q: \mathscr{D}\left(A^{*}\right) \rightarrow \mathscr{D}(A), \quad P: \mathscr{D}(A) \rightarrow \mathscr{D}\left(A^{*}\right),
$$

$\hat{Q}: \mathscr{H} \rightarrow \mathscr{D}(A), \quad \hat{P}: \mathscr{H} \rightarrow \mathscr{D}\left(A^{*}\right)$,

$(3.14 \mathrm{a}, \mathrm{b}, \mathrm{c})^{5}$

$\rho(\hat{Q})=\rho(\hat{P})=\rho(\hat{Q} \hat{P})=n_{c}$,

$$
\begin{aligned}
& 0=(A-\tau Q \bar{\Sigma}) Q+Q(A-\tau Q \bar{\Sigma})^{*}+V_{1}+\tau Q \bar{\Sigma} Q \tau^{*}, \\
& 0=(A-\Sigma P \tau)^{*} P+P(A-\Sigma P \tau)+R_{1}+\tau^{*} P \Sigma P \tau, \\
& 0=\left[(A-\Sigma P) \hat{Q}+\hat{Q}(A-\Sigma P)^{*}+Q \bar{\Sigma} Q\right] \tau^{*}, \\
& 0=\left[(A-Q \bar{\Sigma})^{*} \hat{P}+\hat{P}(A-Q \bar{\Sigma})+P \Sigma P\right] \tau .
\end{aligned}
$$

The content of the Main Theorem is clearly a set of necessary conditions which characterize the optimal steady-state fixed-order dynamic compensator when it exists. These necessary conditions consist of a system of four operator equations including a pair of modified Riccati equations (3.15) and (3.16) and a pair of modified Lyapunov equations (3.17) and (3.18). The salient feature of these four equations is the coupling by the operator $\tau \in \mathscr{B}(\mathscr{H})$ which, because of (3.8), is idempotent, i.e., $\tau^{2}=\tau$. In general, $\tau$ is an oblique projection and not an orthogonal projection since there is no requirement that $\tau$ be selfadjoint. Additional features of the Main Theorem will be discussed in the remainder of this section. For convenience, let $G, M, \Gamma, \tau, Q, P, \hat{Q}$ and $\hat{P}$ be as given by the Main Theorem and define $\Lambda \triangleq \operatorname{diag}\left(\lambda_{1}, \cdots, \lambda_{n_{c}}\right)$, where $\lambda_{1} \geqq \lambda_{2} \geqq \cdots \geqq$ $\lambda_{n_{\mathrm{c}}}>0$ are the eigenvalues of $M$.

We begin by noting that if $x_{c}$ is replaced by $S x_{c}$, where $S \in \mathbb{R}^{n_{c} \times n_{c}}$ is invertible, then an "equivalent" compensator is obtained with $\left(A_{c}, B_{c}, C_{c}\right)$ replaced by $\left(S A_{c} S^{-1}, S B_{c}, C_{c} S^{-1}\right)$.

Proposition 3.1. Let $\left(A_{c}, B_{c}, C_{c}\right) \in \mathscr{A}_{+}$. If $S \in \mathbb{R}^{n_{c} \times n_{c}} \quad$ is invertible then $\left(S A_{c} S^{-1}, S B_{c}, C_{c} S^{-1}\right) \in \mathscr{A}_{+}$and

$$
J\left(A_{c}, B_{c}, C_{c}\right)=J\left(S A_{c} S^{-1}, S B_{c}, C_{c} S^{-1}\right) .
$$

Proof. Although the result is obvious from system-theoretic arguments, we shall prove it analytically by utilizing elements of the development in $\S 4$. Define

$$
\tilde{S} \triangleq\left[\begin{array}{cc}
I_{\infty} & 0 \\
0 & S
\end{array}\right] \in \mathscr{B}(\tilde{\mathscr{H}})
$$

and note that replacing $\left(A_{c}, B_{c}, C_{c}\right)$ by $\left(S A_{c} S^{-1}, S B_{c}, C_{c} S^{-1}\right)$ is equivalent to replacing $\tilde{A}, \tilde{V}$ and $\tilde{R}$ by $\tilde{S} \tilde{A} \tilde{S}^{-1}, \tilde{S} \tilde{V} \tilde{S}^{*}$ and $\tilde{S}^{-*} \tilde{R} \tilde{S}^{-1}$, respectively. If $\alpha, \beta>0$ satisfy (3.6) then a straightforward application of the Hille-Yosida theorem [57, pp. 153-5] shows that

\footnotetext{
${ }^{5}(3.14 \mathrm{a})$ refers to $\rho(\hat{Q})=n_{c}$, etc.
} 
the strongly continuous semigroup generated by $\tilde{S} \tilde{A} \tilde{S}^{-1}$ satisfies $\left\|e^{\tilde{S} \tilde{A} \tilde{S}^{-1} t}\right\| \leqq$ $\|\tilde{S}\|\left\|\tilde{S}^{-1}\right\| \alpha e^{-\beta t}$, which proves the first assertion. Since $\tilde{S} e^{\tilde{A} t} \tilde{S}^{-1}, t \geqq 0$, is also a strongly continuous semigroup with generator $\tilde{S} \tilde{A} \tilde{S}^{-1}$, it follows that $\tilde{S} e^{\tilde{A} t} \tilde{S}^{-1}=e^{\tilde{\tilde{S} \tilde{A}} \tilde{S}^{-1} t}$. Hence

$$
\int_{0}^{\infty} e^{\tilde{S} \tilde{A} \tilde{S}^{-1} t}\left(\tilde{S} \tilde{V} \tilde{S}^{*}\right) e^{\left(\tilde{S} \tilde{A} \tilde{S}^{-1}\right)^{*} t} d t=\tilde{S} \tilde{Q} \tilde{S}^{*}
$$

and (3.19) follows from $\operatorname{tr} \tilde{Q} \tilde{R}=\operatorname{tr}\left(\tilde{S} \tilde{Q} \tilde{S}^{*}\right)\left(\tilde{S}^{-*} \tilde{R} \tilde{S}^{-1}\right)$.

In view of Proposition 3.1 one would expect the Main Theorem to apply also to $\left(S A_{c} S^{-1}, S B_{c}, C_{c} S^{-1}\right)$. Indeed, it may be noted that no claim was made as to the uniqueness of the $(G, M, \Gamma)$-factorization of $\hat{Q} \hat{P}$ used to determine $A_{c}, B_{c}$ and $C_{c}$ in (3.9)-(3.11). These observations are reconciled by the following result which shows that a transformation of the compensator state basis corresponds to the alternative factorization $\hat{Q} \hat{P}=\left(S^{-T} G\right)^{T}\left(S M S^{-1}\right)(S \Gamma)$ and, moreover, that all $(G, M, \Gamma)$-factorizations of $\hat{Q} \hat{P}$ are related by a nonsingular transformation. Note that $\tau$ remains invariant over the class of factorizations.

Proposition 3.2. If $S \in \mathbb{R}^{n_{c} \times n_{c}}$ is invertible then $\bar{G} \triangleq S^{-T} G, \bar{\Gamma} \triangleq S \Gamma$ and $\bar{M} \triangleq S M S^{-1}$ satisfy

$$
\begin{aligned}
& \hat{Q} \hat{P}=\bar{G}^{*} \bar{M} \bar{\Gamma}, \\
& \bar{\Gamma} \bar{G}^{*}=I_{n_{\dot{c}}}
\end{aligned}
$$

Conversely, if $\bar{G}, \bar{\Gamma} \in \mathscr{B}\left(\mathscr{H}, \mathbb{R}^{n_{c}}\right)$ and invertible $\bar{M} \in \mathbb{R}^{n_{c} \times n_{c}}$ satisfy $(3.7)^{\prime}$ and $(3.8)^{\prime}$, then there exists invertible $S \in \mathbb{R}^{n_{c} \times n_{c}}$ such that $\bar{G}=S^{-T} G, \bar{\Gamma}=S \Gamma$ and $\bar{M}=S M S^{-1}$.

Proof. The first part of the proposition is immediate. The second part follows by taking $S \triangleq \bar{M}^{-1} \bar{\Gamma} G^{*} M^{-1}$, noting $S^{-1}=M \bar{\Gamma} \bar{G}^{*} \bar{M}^{-1}$ and using the identities $\bar{\Gamma} G^{*} M \Gamma \bar{G}^{*}=\bar{M}$ and $M \Gamma \bar{G}^{*}=\Gamma \bar{G}^{*} \bar{M}$.

The next result shows that there exists a similarity transformation which simultaneously diagonalizes $\hat{Q} \hat{P}$ and $\tau$.

Proposition 3.3. There exists invertible $\Phi \in \mathscr{B}(\mathscr{H})$ such that

$$
\begin{array}{ll}
\hat{Q}=\Phi^{-1}\left[\begin{array}{cc}
\Lambda_{\hat{Q}} & 0 \\
0 & 0
\end{array}\right] \Phi^{-*}, & \hat{P}=\Phi^{*}\left[\begin{array}{cc}
\Lambda_{\hat{P}} & 0 \\
0 & 0
\end{array}\right] \Phi, \\
\hat{Q} \hat{P}=\Phi^{-1}\left[\begin{array}{ll}
\Lambda & 0 \\
0 & 0
\end{array}\right] \Phi, & \tau=\Phi^{-1}\left[\begin{array}{cc}
I_{n_{c}} & 0 \\
0 & 0
\end{array}\right] \Phi,
\end{array}
$$

where $\Lambda_{\hat{Q}}, \Lambda_{\hat{P}} \in \mathbb{R}^{n_{c} \times n_{c}}$ are positive diagonal and $\Lambda_{\hat{Q}} \Lambda_{\hat{P}}=\Lambda$. Consequently,

$$
\hat{Q}=\tau \hat{Q}, \quad \hat{P}=\hat{P} \tau .
$$

Proof. Proceeding as in the proof of Lemma 3.1, choose an orthonormal basis for $\mathscr{H}$ with respect to which

$$
\hat{Q}=\left[\begin{array}{cc}
\hat{Q}_{1} & 0 \\
0 & 0
\end{array}\right] \text { and } \hat{P}=\left[\begin{array}{cc}
\hat{P}_{1} & 0 \\
0 & 0
\end{array}\right],
$$

where $\hat{Q}_{1}, \hat{P}_{1} \in \mathbb{R}^{r \times r}$ are nonnegative definite. By [55, Thm. 6.2.5, p. 123], there exists invertible $\Psi \in \mathbb{R}^{r \times r}$ such that $\tilde{\Lambda}_{\hat{Q}} \triangleq \Psi \hat{Q}_{1} \Psi^{T}$ and $\tilde{\Lambda}_{\hat{P}}=\Psi^{-T} \hat{P}_{1} \Psi^{-1}$ are nonnegative diagonal. Because of (3.14), it is clear that $\Psi$ can be chosen so that

$$
\tilde{\Lambda}_{\hat{Q}}=\left[\begin{array}{cc}
\Lambda_{\hat{Q}} & 0 \\
0 & 0
\end{array}\right] \text { and } \tilde{\Lambda}_{\hat{P}}=\left[\begin{array}{cc}
\Lambda_{\hat{P}} & 0 \\
0 & 0
\end{array}\right] \text {, }
$$


where $\Lambda_{\hat{Q}}, \Lambda_{\hat{P}} \in \mathbb{R}^{n_{c} \times n_{c}}$ are positive diagonal. Thus (3.20) holds with

$$
\Phi \triangleq\left[\begin{array}{cc}
\Psi & 0 \\
0 & I_{\infty}
\end{array}\right] .
$$

From (3.20) it follows that

$$
\hat{Q} \hat{P}=\Phi^{-1}\left[\begin{array}{cc}
\Lambda_{Q} \Lambda_{P} & 0 \\
0 & 0
\end{array}\right] \Phi .
$$

Now define $\bar{G}=\left[I_{n_{c}} 0\right] \Phi^{-*}, \bar{M}=\Lambda_{\hat{Q}} \Lambda_{\hat{P}}$ and $\bar{\Gamma}=\left[I_{n_{c}} 0\right] \Phi$ so that $(3.7)^{\prime}$ and $(3.8)^{\prime}$ are satisfied. By the second part of Proposition 3.2 there exists invertible $S \in \mathbb{R}^{n_{c} \times n_{c}}$ such that $G=S^{T} \bar{G}, M=S^{-1} \bar{M} S$ and $\Gamma=S^{-1} \bar{\Gamma}$. Since $M$ and $\bar{M}$ have the same eigenvalues, $\bar{M}=\Lambda$ (modulo an ordering of the diagonal elements) and thus (3.21a) holds. Finally, (3.21b) follows from

$$
\tau=G^{*} \Gamma=\bar{G}^{*} \bar{\Gamma}=\Phi^{-1}\left[\begin{array}{cc}
I_{n_{c}} & 0 \\
0 & 0
\end{array}\right] \Phi
$$
of $\hat{Q} \hat{P}$.

Remark 3.1. Proposition 3.3 shows that $\lambda_{1}, \cdots, \lambda_{n_{c}}$ are the positive eigenvalues

Remark 3.2. The simultaneous diagonalization in (3.20) has been effected by a contragredient transformation [55], [58]. For applications of this type of transformation to model reduction and realization problems see [12], [59]-[61]. Simultaneous diagonalization of operators is discussed in [53, p. 181].

The following result validates the precise handling of the unbounded operator $A$ in (3.9), (3.17) and (3.18).

Proposition 3.4. The following relations hold:

$(3.23 \mathrm{a}, \mathrm{b}, \mathrm{c})$

$(3.24 \mathrm{a}, \mathrm{b})$

$(3.25 \mathrm{a}, \mathrm{b})$

$$
\begin{aligned}
& \rho(G)=\rho(\Gamma)=\rho(\tau)=n_{c}, \\
& \tau: \mathscr{H} \rightarrow \mathscr{D}(A), \quad \tau^{*}: \mathscr{H} \rightarrow \mathscr{D}\left(A^{*}\right), \\
& G^{*}: \mathbb{R}^{n_{\mathrm{c}}} \rightarrow \mathscr{D}(A), \quad \Gamma^{*}: \mathbb{R}^{n_{\mathrm{c}}} \rightarrow \mathscr{D}\left(A^{*}\right) .
\end{aligned}
$$

Proof. From (3.8) and (2.1) it follows that $n_{c}=\rho\left(\Gamma G^{*}\right) \leqq \min \left\{\rho(\Gamma), \rho\left(G^{*}\right)\right\}$. Since $\rho(\Gamma) \leqq n_{c}, \rho(G)=\rho\left(G^{*}\right)$ and $\rho(G) \leqq n_{c}$, (3.23a) and (3.23b) hold. To show (3.23c) either note $(3.21 \mathrm{~b})$ or use $(3.14 \mathrm{a})$ and $(3.22)$ to obtain

$$
n_{c}=\rho(\hat{Q})=\rho(\tau \hat{Q}) \leqq \rho(\tau)=\rho\left(G^{*} \Gamma\right) \leqq \rho(\Gamma)=n_{c} .
$$

To prove (3.24a) note that (3.22a) implies $\mathscr{R}(\hat{Q}) \subset \mathscr{R}(\tau)$ and thus $\rho(\hat{Q})=\rho(\tau)$ implies $\mathscr{R}(\hat{Q})=\mathscr{R}(\tau)$, and similarly for (3.24b). Finally, (3.25) follows from (3.23), (3.24), the definition $\tau=G^{*} \Gamma$ and the fact that $\tau^{*}=\Gamma^{*} G$.

Since the domain of $A$ may not be all of $\mathscr{H}$, expressions involving $A$ require special interpretation. First note that because of the range condition (3.25a), the expression (3.9) indeed represents an $n_{c} \times n_{c}$ matrix (see, e.g., [6, p. 80]). Similarly, because of (3.25b), $A_{c}^{T}$ is given by

$$
A_{c}^{T}=G\left(A^{*}-\bar{\Sigma} Q-P \Sigma\right) \Gamma^{*} .
$$

With regard to (3.15), note that because of (3.12a), the right-hand side of (3.15) is a linear operator with domain $\mathscr{D}\left(A^{*}\right)$. Since $\Theta \triangleq-\tau Q \bar{\Sigma} Q-Q \bar{\Sigma} Q \tau^{*}+V_{1}+\tau Q \bar{\Sigma} Q \tau^{*}$ is continuous on $\mathscr{D}\left(A^{*}\right), A Q+Q A^{*}$ has a continuous extension on $\mathscr{H}$ given precisely by $-\Theta$. Similar remarks apply to (3.16). Analogous domain conditions were obtained in [5] for a deterministic infinite-dimensional linear-quadratic control problem with 
full-state feedback. Finally, because of (3.24) the right-hand sides of (3.17) and (3.18) denote bounded linear operators on all of $\mathscr{H}$.

It is useful to present an alternative form of the optimal projection equations (3.15)-(3.18). For convenience define the notation

$$
\tau_{\perp} \triangleq I_{\mathscr{H}}-\tau
$$

Proposition 3.5. Equations (3.15)-(3.18) are equivalent, respectively, to

$$
\begin{aligned}
& 0=A Q+Q A^{*}+V_{1}-Q \bar{\Sigma} Q+\tau_{\perp} Q \bar{\Sigma} Q \tau_{\perp}^{*}, \\
& 0=A^{*} P+P A+R_{1}-P \Sigma P+\tau_{\perp}^{*} P \Sigma P \tau_{\perp}, \\
& 0=(A-\Sigma P) \hat{Q}+\hat{Q}(A-\Sigma P)^{*}+Q \bar{\Sigma} Q-\tau_{\perp} Q \bar{\Sigma} Q \tau_{\perp}^{*}, \\
& 0=(A-Q \bar{\Sigma})^{*} \hat{P}+\hat{P}(A-Q \bar{\Sigma})+P \Sigma P-\tau_{\perp}^{*} P \Sigma P \tau_{\perp} .
\end{aligned}
$$

Proof. The equivalence of (3.27) and (3.28) to (3.15) and (3.16) is immediate. Using (3.22a) in the form $\hat{Q}=\hat{Q} \tau^{*}$, we obtain $(3.17)=(3.29) \tau^{*}$. Conversely, from (3.22a) and $[(A-\Sigma P) \hat{Q}]^{*}=\hat{Q}(A-\Sigma P)^{*}$ (see, e.g., [6, p. 80]) it follows that $(3.29)=$ $(3.17)+(3.17)^{*}-\tau(3.17)$. Similarly, (3.18) and (3.30) are equivalent.

The form of the optimal projection equations (3.27)-(3.30) helps demonstrate the relationship between the Main Theorem and the classical $L Q G$ result when $\operatorname{dim} \mathscr{H}=n<$ $\infty$. In this case we need only note that the $(G, M, \Gamma)$-factorization of $\hat{Q} \hat{P}$ in the "full-order" case $n_{c}=n$ is given by $G=\Gamma=I_{n}$ and $M=\hat{Q} \hat{P}$. Since $\tau=I_{n}$, and thus $\tau_{\perp}=0,(3.27)$ and (3.28) reduce to the standard observer and regulator Riccati equations and (3.9)-(3.11) yield the usual $L Q G$ expressions. Furthermore, note that in the full-order case

$$
A_{c}=A+B C_{c}-B_{c} C
$$

and (3.29) and (3.31) can be written as

$$
\begin{aligned}
& 0=\left(A_{c}+B_{c} C\right) \hat{Q}+\hat{Q}\left(A_{c}+B_{c} C\right)^{T}+B_{c} V_{2} B_{c}^{T}, \\
& 0=\left(A_{c}-B C_{c}\right)^{T} \hat{P}+\hat{P}\left(A_{c}-B C_{c}\right)+C_{c}^{T} R_{2} C_{c} .
\end{aligned}
$$

Since, as is well known, the stability of $\tilde{A}$ corresponds to the stability of $A+B C_{c}=$ $A_{c}+B_{c} C$ and $A-B_{c} C=A_{c}-B C_{c}$, it follows from standard results (e.g., [62, pp. 48, 277]) that the positive-definiteness conditions $(3.14 \mathrm{a}, \mathrm{b})$ are equivalent to the assumption that $\left(A_{c}, B_{c}, C_{c}\right)$ is controllable and observable.

To obtain a geometric intepretation of the optimal projection we introduce the quasi-full-state estimate

$$
\hat{x}(t) \triangleq G^{*} x_{c}(t) \in \mathscr{H}
$$

so that $\tau \hat{x}(t)=\hat{x}(t)$ and $x_{c}(t)=\Gamma \hat{x}(t)$. Now, the closed-loop system (3.1)-(3.4) can be written as

$$
\begin{aligned}
& \dot{x}(t)=A x(t)-B \hat{C}_{c} \tau \hat{x}(t)+H_{1} w(t), \\
& \dot{\hat{x}}(t)=\tau\left(A+B \hat{C}_{c}-\hat{B}_{c} C\right) \tau \hat{x}(t)+\tau \hat{B}_{c}\left(C x(t)+H_{2} w(t)\right),
\end{aligned}
$$

where (3.35) is interpreted in the sense of (3.34) since $\hat{x}(t) \in \mathscr{H}$ and where

$$
\hat{B}_{c} \triangleq Q C^{*} V_{2}^{-1}, \quad \hat{C}_{c} \triangleq-R_{2}^{-1} B^{*} P .
$$

It can thus be seen that the geometric structure of the quasi-full-order compensator is entirely dictated by the projection $\tau$. In particular, control inputs $\tau \hat{x}(t)$ determined by 
(3.35) are contained in $\mathscr{R}(\tau)$ and sensor inputs $\tau \hat{B}_{c} y(t)$ are annihilated unless they are contained in $[\mathcal{N}(\tau)]^{\perp}=\mathscr{R}\left(\tau^{*}\right)$. Consequently, $\mathscr{R}(\tau)$ and $\mathscr{R}\left(\tau^{*}\right)$ are the control and observation subspaces, respectively, of the compensator. Since $\tau$ is not necessarily an orthogonal projection, these (finite-dimensional) subspaces may be different.

From the form of (3.35) it is tempting to suggest that the optimal fixed-order dynamic compensator can be obtained by projecting the full-order (infinitedimensional) $L Q G$ compensator. However, this is generally impossible for the following simple reason. Although the expressions for $A_{c}, B_{c}$ and $C_{c}$ in (3.9)-(3.11) have the form of a projection of the full-order $L Q G$ compensator, the operators $Q$ and $P$ in (3.9)-(3.11) are not the solutions of the usual $L Q G$ Riccati equations but instead must be obtained by simultaneously solving all four coupled equations (3.15)-(3.18). This observation reinforces the statement made in $\S 1$ that the optimal fixed-order dynamic compensator cannot in general be obtained by $L Q G$ followed by closed-loop controller reduction as in [14] and [15].

We now give an explicit characterization of the optimal projection in terms of $\hat{Q}$ and $\hat{P}$. Since $\hat{Q} \hat{P}$ has finite rank, its Drazin inverse $(\hat{Q} \hat{P})^{D}$ exists (see $[63$, Thm. 6, p. $108]$ ) and, since $(\hat{Q} \hat{P})^{2}=G^{*} M^{2} \Gamma$, and hence $\rho(\hat{Q} \hat{P})^{2}=\rho(\hat{Q} \hat{P})$, the "index" of $\hat{Q} \hat{P}$ (see [63], [64]) is 1 . In this case the Drazin inverse is traditionally called the group inverse and is denoted by $(\hat{Q} \hat{P})^{\#}$ (see, e.g., [64, p. 124] or [65]).

Proposition 3.6. The optimal projection $\tau$ is given by

$$
\tau=\hat{Q} \hat{P}(\hat{Q} \hat{P})^{\#} .
$$

Proof. It is easy to verify that the conditions characterizing the Drazin inverse [63] for the case that $\hat{Q} \hat{P}$ has index 1 are satisfied by $G^{*} M^{-1} \Gamma$. Hence $(\hat{Q} \hat{P})^{\#}=G^{*} M^{-1} \Gamma$ and (3.8) implies (3.36).

We now give an alternative characterization of the optimal projection by introducing the following notation from [51, p. 73]. For $\phi, \psi \in \mathscr{H}$ define the operator $\phi \otimes \psi \in$ $\mathscr{B}(\mathscr{H})$ by

$$
(\phi \otimes \psi) x \triangleq\langle x, \phi\rangle \psi, \quad x \in \mathscr{H},
$$

and note that $\rho(\phi \otimes \psi)=1$ if $\phi$ and $\psi$ are both nonzero and $(\phi \otimes \psi)^{*}=\psi \otimes \phi$. Using this notation, (3.21a) can be written as

$$
\Phi \hat{Q} \hat{P} \Phi^{-1}=\sum_{i=1}^{n_{c}} \lambda_{i} \xi_{i} \otimes \xi_{i},
$$

where $\left\{\xi_{i}\right\}_{i=1}^{\infty}$ is an orthonormal basis for $\mathscr{H}$. In terms of the Riesz bases (see e.g., [52, p. 309])

$$
\phi_{i} \triangleq \Phi^{*} \xi_{i}, \quad \psi_{i} \triangleq \Phi^{-1} \xi_{i}, \quad i=1,2, \cdots,
$$

(3.37) is equivalent to

$$
\hat{Q} \hat{P}=\sum_{i=1}^{n_{c}} \lambda_{i} \phi_{i} \otimes \psi_{i}
$$

which can be regarded as a specialized spectral decomposition of a semisimple operator. We emphasize that, in contrast to the singular value decomposition for compact nonnormal operators (see, e.g., [50, p. 261]), the $\lambda_{i}$ in (3.38) are eigenvalues of $\hat{Q} \hat{P}$ (see Remark 3.1), not singular values. Moreover, although $\left\{\phi_{i}\right\}_{i=1}^{\infty}$ and $\left\{\psi_{i}\right\}_{i=1}^{\infty}$ are bases for $\mathscr{H}$, they are not necessarily orthogonal. They are, however, biorthonormal, i.e., $\left\langle\phi_{i}, \psi_{j}\right\rangle=\delta_{i j}$, and hence $\phi_{i} \otimes \psi_{i}$ is a rank-one projection and $\left(\phi_{i} \otimes \psi_{i}\right)\left(\phi_{j} \otimes \psi_{j}\right)=0, i \neq j$. 
Since $\tau$ is a rank- $n_{c}$ projection, it is not surprising that $\tau$ is given precisely by

$$
\tau=\sum_{i=1}^{n_{c}} \phi_{i} \otimes \psi_{i}
$$

The following result summarizes the above observations.

PRoposition 3.7. There exist biorthonormal linearly independent sets $\left\{\psi_{i}\right\}_{i=1}^{n_{c}} \subset$ $\mathscr{D}(A)$ and $\left\{\phi_{i}\right\}_{i=1}^{n_{c}} \subset \mathscr{D}\left(A^{*}\right)$ such that (3.38) and (3.39) hold. Furthermore, if the $(G, M, \Gamma)$-factorization of $\hat{Q} \hat{P}$ is chosen such that $M=\Lambda$, then, for all $x \in \mathscr{H}$,

$$
\begin{aligned}
& G x=\left(\left\langle x, \psi_{1}\right\rangle, \cdots,\left\langle x, \psi_{n_{c}}\right\rangle\right)^{T}, \\
& \Gamma x=\left(\left\langle x, \phi_{1}\right\rangle, \cdots,\left\langle x, \phi_{n_{c}}\right\rangle\right)^{T} .
\end{aligned}
$$

Remark 3.3. Note that $\hat{P} \hat{Q}$ and $\tau^{*}$ are given by

$$
\hat{P} \hat{Q}=\sum_{i=1}^{n_{c}} \lambda_{i} \psi_{i} \otimes \phi_{i}, \quad \tau^{*}=\sum_{i=1}^{n_{c}} \psi_{i} \otimes \phi_{i},
$$

and, for all $y \triangleq\left(y_{1}, \cdots, y_{n_{c}}\right)^{T} \in \mathbb{R}^{n_{c}}, G^{*}$ and $\Gamma^{*}$ satisfy

$$
G^{*} y=\sum_{i=1}^{n_{c}} y_{i} \psi_{i}, \quad \Gamma^{*} y=\sum_{i=1}^{n_{c}} y_{i} \phi_{i}
$$

4. Proof of the Main Theorem. We state and prove a series of lemmas which allow us to compute the Frechet derivatives of $J$ with respect to $A_{c}, B_{c}$ and $C_{c}$. Requiring that these derivatives vanish leads to the necessary conditions in their "primitive" form. A transformation of variables then leads to the form of the necessary conditions (3.9)-(3.18).

Let "u-lim" denote the uniform limit (i.e., limit in operator norm) for bounded linear operators $[50$, p. 150$]$ and, for strongly continuous $S(t) \in \mathscr{B}(\mathscr{H}), t \geqq 0$, interpret the strong integral $\int_{t_{1}}^{t_{2}} S(t) d t$ according to $\int_{t_{1}}^{t_{2}} S(t) z d t, z \in \mathscr{H}[50$, p. 152]. Also recall the standard fact $\left[6\right.$, p. 186] that $\left(e^{A t}\right)^{*}=e^{A^{*} t}$ and similarly for $\tilde{A}$. Throughout this section let $\left(A_{c}, B_{c}, C_{c}\right) \in \mathscr{A}_{+}$and let $\alpha, \beta>0$ satisfy (3.6).

To begin, note that the closed-loop system (3.1)-(3.4) can be written as

$$
\tilde{x}(t)=\tilde{A} \tilde{x}(t)+\tilde{H} w(t),
$$

where

$$
\tilde{H} \triangleq\left[\begin{array}{c}
H_{1} \\
B_{c} H_{2}
\end{array}\right] \in \mathscr{B}_{2}\left(\mathscr{H}^{\prime} \oplus \mathbb{R}^{l}\right) .
$$

For convenience define the nonnegative-definite operator

$$
\tilde{V} \triangleq \tilde{H} \tilde{H}^{*}=\left[\begin{array}{cc}
V_{1} & 0 \\
0 & B_{c} V_{2} B_{c}^{T}
\end{array}\right] \in \mathscr{B}_{1}(\tilde{\mathscr{H}}) .
$$

In terms of the augmented state $\tilde{x}(t)$, the performance criterion (3.5) becomes

$$
J\left(A_{c}, B_{c}, C_{c}\right)=\lim _{t \rightarrow \infty} \mathbb{E}\langle\tilde{R} \tilde{x}(t), \tilde{x}(t)\rangle,
$$

where the nonnegative-definite operator $\tilde{R}$ is defined by

$$
\tilde{R} \triangleq\left[\begin{array}{cc}
R_{1} & 0 \\
0 & C_{c}^{T} R_{2} C_{c}
\end{array}\right] \in \mathscr{B}_{1}(\tilde{\mathscr{H}}) .
$$


To write (4.2) in terms of the covariance of $\tilde{x}(t)$, recall $[6$, p. 308] that the covariance " $\mathbb{E}\left[(\xi-\mathbb{E} \xi)(\xi-\mathbb{E} \xi)^{*}\right]$ " of a Hilbert-space-valued weak random variable $\xi$ is defined to be the nonnegative-definite operator $S$ which satisfies

$$
\langle S y, z\rangle=\mathbb{E}\langle\xi-\mathbb{E} \xi, y\rangle\langle\xi-\mathbb{E} \xi, z\rangle
$$

for all $y, z$ in the Hilbert space. Hence define [6, p. 317]

$$
\tilde{Q}(t) \triangleq \mathbb{E}\left[(\tilde{x}(t)-\mathbb{E} \tilde{x}(t))(\tilde{x}(t)-\mathbb{E} \tilde{x}(t))^{*}\right] .
$$

LEMMA 4.1. $\tilde{Q} \triangleq u-\lim _{t \rightarrow \infty} \tilde{Q}(t)$ exists and is given by

$$
\tilde{Q}=\int_{0}^{\infty} e^{\tilde{A} t} \tilde{V} e^{\tilde{A}^{*} t} d t
$$

Furthermore,

$$
J\left(A_{c}, B_{c}, C_{c}\right)=\operatorname{tr} \tilde{Q} \tilde{R} .
$$

Proof. First compute (as in [6, p. 317])

$$
\begin{aligned}
& \langle\tilde{Q}(t) \tilde{y}, \tilde{z}\rangle=\mathbb{E}\left\langle\tilde{x}(t)-e^{\tilde{A} t} \mathbb{E} \tilde{x}(0), \tilde{y}\right\rangle\left\langle\tilde{x}(t)-e^{\tilde{A} t} \mathbb{E} \tilde{x}(0), \tilde{z}\right\rangle \\
& =\mathbb{E}\left\langle\int_{0}^{t} e^{\tilde{A}(t-s)} \tilde{H} \tilde{w}(s) d s, \tilde{y}\right\rangle\left\langle\int_{0}^{t} e^{\tilde{A}(t-\sigma)} \tilde{H} \tilde{w}(\sigma) d \sigma, \tilde{z}\right\rangle \\
& +\left\langle\tilde{Q}(0) e^{\tilde{A}^{*} t} \tilde{y}, e^{\tilde{A}^{*} t} \tilde{z}\right\rangle \\
& =\mathbb{E} \int_{0}^{t} \int_{0}^{t}\left\langle\tilde{w}(s), \tilde{H}^{*} e^{\tilde{A}^{*}(t-s)} \tilde{y}\right\rangle\left\langle\tilde{w}(\sigma), \tilde{H}^{*} e^{\tilde{A}^{*}(t-\sigma)} \tilde{z}\right\rangle d s d \sigma \\
& +\left\langle e^{\tilde{A} t} \tilde{Q}(0) e^{\tilde{A}^{*} t} \tilde{y}, \tilde{z}\right\rangle \\
& =\int_{0}^{t}\left\langle e^{\tilde{A}(t-s)} \tilde{V} e^{\tilde{A}^{*}(t-s)} \tilde{y}, \tilde{z}\right\rangle d s+\left\langle e^{\tilde{A} t} \tilde{Q}(0) e^{\tilde{A}^{*} t} \tilde{y}, \tilde{z}\right\rangle,
\end{aligned}
$$

which shows that $\tilde{Q}(t)$ is given by

$$
\tilde{Q}(t)=e^{\tilde{A} t} \tilde{Q}(0) e^{\tilde{A}^{*} t}+\int_{0}^{t} e^{\tilde{A} s} \tilde{V} e^{\tilde{A}^{*} s} d s .
$$

Clearly, (4.3) makes sense as a strong integral since

$$
\|\tilde{Q}\| \leqq \int_{0}^{\infty}\left\|e^{\tilde{A} t} \tilde{V} e^{\tilde{A}^{*} t}\right\| d t \leqq \alpha^{2}\|\tilde{V}\| \int_{0}^{\infty} e^{-2 \beta t} d t<\infty
$$

To demonstrate uniform convergence it need only be noted that

$$
\begin{aligned}
\|\tilde{Q}-\tilde{Q}(t)\| & =\sup _{\|\tilde{y}\|=1}\|(\tilde{Q}-\tilde{Q}(t)) \tilde{y}\| \\
& =\sup _{\|\tilde{y}\|=1}\left\|\int_{t}^{\infty} e^{\tilde{A} s} \tilde{V} e^{\tilde{A}^{*} s} \tilde{y} d s-e^{\tilde{A} t} \tilde{Q}(0) e^{\tilde{A}^{*} t} \tilde{y}\right\| \\
& \leqq \int_{t}^{\infty}\left\|e^{\tilde{A} s} \tilde{V} e^{\tilde{A}^{*} s}\right\| d s+\left\|e^{\tilde{A} t} \tilde{Q}(0) e^{\tilde{A}^{*} t}\right\| \\
& \leqq \frac{1}{2} \alpha^{2}\|\tilde{V}\| \beta^{-1} e^{-2 \beta t}+\|\tilde{Q}(0)\| e^{-2 \beta t}
\end{aligned}
$$


Next, let $\left\{\phi_{i}\right\}_{i=1}^{\infty}$ be an orthonormal basis for $\tilde{\mathscr{H}}$ and use Parseval's equality to obtain

$$
J\left(A_{c}, B_{c}, C_{c}\right)=\lim _{t \rightarrow \infty} \mathbb{E}\left\|\tilde{R}^{1 / 2} \tilde{x}(t)\right\|^{2}=\lim _{t \rightarrow \infty} \mathbb{E} \sum_{i=1}^{\infty}\left\langle\tilde{R}^{1 / 2} \tilde{x}(t), \phi_{i}\right\rangle^{2}
$$

Since

$$
f_{n}(t) \triangleq \sum_{i=1}^{n}\left\langle\tilde{R}^{1 / 2} \tilde{x}(t), \phi_{i}\right\rangle^{2}, \quad t \geqq 0,
$$

is nonnegative for each $n$ and is increasing in $n$ for each $t$ with limit $\langle\tilde{R} \tilde{x}(t), \tilde{x}(t)\rangle$, monotone convergence permits expectation-limit interchange. Hence using $\mathbb{E} \tilde{x}(t)=$ $e^{\tilde{A} t} \mathbb{E} \tilde{x}(0)$ we have

$$
\begin{aligned}
J\left(A_{c}, B_{c}, C_{c}\right) & =\lim _{t \rightarrow \infty} \sum_{i=1}^{\infty} \mathbb{E}\left\langle\tilde{x}(t), \tilde{R}^{1 / 2} \phi_{i}\right\rangle^{2} \\
& =\lim _{t \rightarrow \infty} \sum_{i=1}^{\infty}\left[\left\langle\tilde{Q}(t) \tilde{R}^{1 / 2} \phi_{i}, \tilde{R}^{1 / 2} \phi_{i}\right\rangle+\left\langle e^{\tilde{A} t} \mathbb{E} \tilde{x}(0), \tilde{R}^{1 / 2} \phi_{i}\right\rangle^{2}\right] \\
& =\lim _{t \rightarrow \infty}\left\{\operatorname{tr}\left[\tilde{R}^{1 / 2} \tilde{Q}(t) \tilde{R}^{1 / 2}\right]+\left\|\tilde{R}^{1 / 2} e^{\tilde{A} t} \mathbb{E} \tilde{x}(0)\right\|^{2}\right\}
\end{aligned}
$$

which by Corollary 2.1 yields (4.4).

We shall also require the "dual" of $\tilde{Q}$ given by

$$
\tilde{P}=\int_{0}^{\infty} e^{\tilde{A}^{*} t} \tilde{R} e^{\tilde{A} t} d t
$$

Since $\tilde{V}$ and $\tilde{R}$ are nonnegative definite it is readily seen that $\tilde{Q}$ and $\tilde{P}$ are also nonnegative definite.

LeMma 4.2. $\tilde{Q}, \tilde{P} \in \mathscr{B}_{1}(\tilde{\mathscr{H}})$.

Proof. It suffices to consider $\tilde{Q}$ only since the situation for $\tilde{P}$ is exactly analogous. Since $\tilde{Q}$ is nonnegative definite, Lemma 2.3 can be used. Letting $\left\{\phi_{i}\right\}_{i=1}^{\infty}$ be an orthonormal basis for $\tilde{\mathscr{H}}$, we have

$$
\begin{aligned}
\operatorname{tr} \tilde{Q} & =\sum_{i=1}^{\infty}\left\langle\tilde{Q} \phi_{i}, \phi_{i}\right\rangle=\sum_{i=1}^{\infty}\left\langle\int_{0}^{\infty} e^{\tilde{A} t} \tilde{V} e^{\tilde{A}^{*} t} \phi_{i} d t, \phi_{i}\right\rangle \\
& =\lim _{n \rightarrow \infty} \int_{0}^{\infty} \sum_{i=1}^{n}\left\langle\tilde{V} e^{\tilde{A}^{*} t} \phi_{i}, e^{\tilde{A}^{*} t} \phi_{i}\right\rangle d t .
\end{aligned}
$$

Let $f_{n}(t)$ denote the above integrand. Since $\tilde{V}$ is nonnegative definite, $\left\{f_{n}(\cdot)\right\}$ is a monotonically increasing sequence of nonnegative functions such that $f_{n}(t) \rightarrow$ $\operatorname{tr} e^{\tilde{A} t} \tilde{V} e^{\tilde{A}^{*} t}, t \geqq 0$. Hence, by monotone convergence and Lemma 2.2,

$$
\begin{aligned}
\operatorname{tr} \tilde{Q} & =\int_{0}^{\infty} \operatorname{tr}\left[e^{\tilde{A} t} \tilde{V} e^{\tilde{A}^{*} t}\right] d t \\
& =\int_{0}^{\infty}\left\|e^{\tilde{A} t} \tilde{V} e^{\tilde{A}^{*} t}\right\|_{1} d t \leqq \alpha^{2}\|\tilde{V}\|_{1} \int_{0}^{\infty} e^{-2 \beta t} d t<\infty
\end{aligned}
$$

LeMma 4.3. With $\tilde{Q}$ and $\tilde{P}$ given by (4.3) and (4.5) it follows that

$$
\operatorname{tr} \tilde{Q} \tilde{R}=\operatorname{tr} \tilde{V} \tilde{P} .
$$


Proof. For any orthonormal basis $\left\{\phi_{i}\right\}_{i=1}^{\infty}$ of $\tilde{\mathscr{H}}$ we have

$$
\begin{aligned}
\operatorname{tr} \tilde{Q} \tilde{R} & =\operatorname{tr} \tilde{R} \tilde{Q}=\sum_{i=1}^{\infty}\left\langle\tilde{R} \int_{0}^{\infty} e^{\tilde{A} t} \tilde{V} e^{\tilde{A}^{*} t} \phi_{i} d t, \phi_{i}\right\rangle \\
& =\lim _{n \rightarrow \infty} \int_{0}^{\infty} \sum_{i=1}^{n}\left\langle\tilde{R} e^{\tilde{A} t} \tilde{V} e^{\tilde{A}^{*} t} \phi_{i}, \phi_{i}\right\rangle d t .
\end{aligned}
$$

Letting $f_{n}(t)$ denote the above integrand it follows that $f_{n}(t) \rightarrow \operatorname{tr} \tilde{R} e^{\tilde{A} t} \tilde{V} e^{\tilde{A}^{*} t}, t \geqq 0$, and

$$
\left|f_{n}(t)\right| \leqq \sum_{i=1}^{\infty}\left|\left\langle e^{\tilde{A} t} \tilde{V} e^{\tilde{A}^{*} t} \phi_{i}, \tilde{R} \phi_{i}\right\rangle\right| \leqq \alpha^{2}\|\tilde{V}\| e^{-2 \beta t} \sum_{i=1}^{\infty}\left\|\tilde{R} \phi_{i}\right\| .
$$

If $\left\{\phi_{i}\right\}_{i=1}^{\infty}$ is chosen to be the set of orthonormal eigenvectors of $\tilde{R}$ then Lemma 2.1 implies $\sum_{i=1}^{\infty}\left\|\tilde{R} \phi_{i}\right\|=\|\tilde{R}\|_{1}$ and thus $\left|f_{n}(t)\right|$ is bounded on $[0, \infty)$ by an integrable function. Hence by dominated convergence,

$$
\operatorname{tr} \tilde{Q} \tilde{R}=\int_{0}^{\infty} \operatorname{tr}\left[\tilde{R} e^{\tilde{A} t} \tilde{V} e^{\tilde{A}^{*} t}\right] d t=\int_{0}^{\infty} \operatorname{tr}\left[e^{\tilde{A}^{*} t} \tilde{R} e^{\tilde{A} t} \tilde{V}\right] d t=\int_{0}^{\infty} \sum_{i=1}^{\infty}\left\langle\tilde{V} \phi_{i}, e^{\tilde{A}^{*} t} \tilde{R} e^{\tilde{A} t}\right\rangle d t
$$

And again using dominated convergence,

$$
\operatorname{tr} \tilde{Q} \tilde{R}=\sum_{i=1}^{\infty} \int_{0}^{\infty}\left\langle\tilde{V} \phi_{i}, e^{\tilde{A}^{*} t} \tilde{R} e^{\tilde{A} t} \phi_{i}\right\rangle d t=\sum_{i=1}^{\infty}\left\langle\tilde{V} \phi_{i}, \int_{0}^{\infty} e^{\tilde{A}^{*} t} \tilde{R} e^{\tilde{A} t} \phi_{i} d t\right\rangle=\operatorname{tr} \tilde{V} \tilde{P} .
$$

The next result is important in that it allows us to treat $\tilde{Q}$ and $\tilde{P}$ as solutions of dual algebraic Lyapunov equations. For a similar result involving groups rather than semigroups see [50, pp. 555-557].

LEMMA 4.4. $\tilde{Q}$ is given by (4.3) if and only if $\tilde{Q} \in \mathscr{B}(\tilde{\mathscr{C}})$ satisfies

$$
\begin{aligned}
& \tilde{Q}: \mathscr{D}\left(\tilde{A}^{*}\right) \rightarrow \mathscr{D}(\tilde{A}), \\
& 0=\tilde{A} \tilde{Q}+\tilde{Q} \tilde{A}^{*}+\tilde{V},
\end{aligned}
$$

where (4.8) holds in the sense discussed in $\S 3$. Furthermore, $\tilde{P}$ is given by (4.5) if and only if $\tilde{\boldsymbol{P}} \in \mathscr{B}(\tilde{\mathscr{H}})$ satisfies

$$
\begin{aligned}
& \tilde{P}: \mathscr{D}(\tilde{A}) \rightarrow \mathscr{D}\left(\tilde{A}^{*}\right), \\
& 0=\tilde{A}^{*} \tilde{P}+\tilde{P} \tilde{A}+\tilde{R} .
\end{aligned}
$$

Proof. We consider $\tilde{Q}$ only. To prove necessity let $t^{\prime}>0$. Then for all $t \in\left[0, t^{\prime}\right)$ and $\tilde{x} \in \mathscr{D}\left(\tilde{A}^{*}\right)$ we can write

$$
\begin{aligned}
e^{\tilde{A} t} \tilde{Q} e^{\tilde{A}^{*} t^{\prime}} \tilde{x} & =\int_{0}^{\infty} e^{\tilde{A}(t+s)} \tilde{V} e^{\tilde{A}^{*}\left(t^{\prime}+s\right)} \tilde{x} d s \\
& =\int_{t}^{\infty} e^{\tilde{A} \sigma} \tilde{V} e^{\tilde{A}^{*} \sigma} e^{\tilde{A}^{*}\left(t^{\prime}-t\right)} \tilde{x} d \sigma .
\end{aligned}
$$

Hence,

$$
\frac{d}{d t} e^{\tilde{A} t} \tilde{Q} e^{\tilde{A}^{*} t^{\prime}} \tilde{x}=-\int_{t}^{\infty} e^{\tilde{A} \sigma} \tilde{V} e^{\tilde{A}^{*} \sigma} e^{\tilde{A}^{*}\left(t^{\prime}-t\right)} \tilde{A^{*}} \tilde{x} d \sigma-e^{\tilde{A} t} \tilde{V} e^{\tilde{A}^{*} t^{\prime}} \tilde{x}
$$

which shows that $e^{\tilde{A} t} \tilde{Q} e^{\tilde{A}^{*} t^{\prime}}$ is strongly differentiable with respect to $t$ for all $t \in\left[0, t^{\prime}\right)$. In particular, setting $t=0$ it follows that $\tilde{Q} e^{\tilde{A}^{*} t^{\prime}} \tilde{x} \in \mathscr{D}(\tilde{A})$ for all $\tilde{x} \in \mathscr{D}\left(\tilde{A}^{*}\right)$ (see, e.g., $[6$, p. 173$]$ or $[50$, p. 485$])$. Performing the differentiation on the left-hand side of 
(4.11) and setting $t=0$ yields

$$
\tilde{A} \tilde{Q} e^{\tilde{A}^{*} t^{\prime}} \tilde{x}=-\int_{0}^{\infty} e^{\tilde{A} \sigma} \tilde{V} e^{\tilde{A}^{*} \sigma} e^{\tilde{A}^{*} t^{\prime}} \tilde{A}^{*} \tilde{x} d \sigma-\tilde{V} e^{\tilde{A}^{*} t^{\prime}} \tilde{x}
$$

Now fix $\tilde{x} \in \mathscr{D}\left(\tilde{A}^{*}\right)$. Then for $\left\{t_{i}\right\}_{i=1}^{\infty}, t_{i}>0, t_{i} \rightarrow 0$, we have

$$
\begin{aligned}
& \tilde{Q} e^{\tilde{A}^{*} t_{i}} \tilde{x} \in \mathscr{D}(\tilde{A}), \quad i=1,2,3, \cdots, \\
& \tilde{Q} e^{\tilde{A}^{*} t_{i}} \tilde{x} \underset{i \rightarrow \infty}{\longrightarrow} \tilde{Q} \tilde{x} .
\end{aligned}
$$

Now consider the sequence $\left\{\tilde{A} \tilde{Q} e^{\tilde{A}^{*} t_{i}} \tilde{x}\right\}_{i=1}^{\infty}$. Letting $t^{\prime}=t_{i}$ in (4.12) and using dominated convergence to interchange limit and integration $\left(\tilde{A}^{*} \tilde{x}\right.$ is a fixed element of $\left.\tilde{\mathscr{C}}\right)$, it follows that

$$
\lim _{i \rightarrow \infty} \tilde{A} \tilde{Q} e^{\tilde{A}^{*} t_{i}} \tilde{x}=-\int_{0}^{\infty} e^{\tilde{A} \sigma} \tilde{V} e^{\tilde{A}^{*} \sigma} \tilde{A} * \tilde{x} d \sigma-\tilde{V} \tilde{x}
$$

Since $\tilde{A}$ is closed, $\tilde{Q} \tilde{x} \in \mathscr{D}(\tilde{A})$. This proves (4.7). Also, since $\tilde{A}$ is closed we have

$$
\lim _{i \rightarrow \infty} \tilde{A} \tilde{Q} e^{\tilde{A}^{*} t_{i}} \tilde{x}=\tilde{A} \tilde{Q} \tilde{x}
$$

which with (4.13) implies

$$
\tilde{A} \tilde{Q} \tilde{x}=-\tilde{Q} \tilde{A}^{*} \tilde{x}-\tilde{V} \tilde{x}
$$

and hence

$$
\left(\tilde{A} \tilde{Q}+\tilde{Q} \tilde{A}^{*}+\tilde{V}\right) \tilde{x}=0, \quad \tilde{x} \in \mathscr{D}\left(\tilde{A}^{*}\right),
$$

as desired.

To prove sufficiency let $\tilde{x} \in \mathscr{D}(\tilde{A})$. Then $e^{\tilde{A}^{*} t} \tilde{x} \in \mathscr{D}\left(\tilde{A}^{*}\right), t \geqq 0$, and hence

$$
\frac{d}{d t} e^{\tilde{A} t} \tilde{Q} e^{\tilde{A}^{*} t} \tilde{x}=e^{\tilde{A} t}\left(\tilde{A} \tilde{Q}+\tilde{Q} \tilde{A}^{*}\right) e^{\tilde{A}^{* t}} \tilde{x}
$$

Thus

$$
e^{\tilde{A} t} \tilde{Q} e^{\tilde{A}^{*} t} \tilde{x}-\tilde{Q} \tilde{x}=\int_{0}^{t} e^{\tilde{A} s}\left(\tilde{A} \tilde{Q}+\tilde{Q} \tilde{A}^{*}\right) e^{\tilde{A}^{*} s} \tilde{x} d s, \quad \tilde{x} \in \mathscr{D}\left(\tilde{A}^{*}\right)
$$

Extending $\tilde{A} \tilde{Q}+\tilde{Q} \tilde{A}^{*}$ to all of $\tilde{\mathscr{H}}$ we obtain

$$
e^{\tilde{A} t} \tilde{Q} e^{\tilde{A}^{*} t} \tilde{x}-\tilde{Q} \tilde{x}=-\int_{0}^{t} e^{\tilde{A} s} \tilde{V} e^{\tilde{A}^{*} s} \tilde{x} d s, \quad \tilde{x} \in \tilde{\mathscr{H}} .
$$

Letting $t \rightarrow \infty$ yields (4.3).

We now introduce some notation which will prove to be most convenient in the following results. For $\left(A_{c}^{\prime}, B_{c}^{\prime}, C_{c}^{\prime}\right) \in \mathbb{R}^{n_{c} \times n_{c}} \times \mathbb{R}^{n_{c} \times l} \times \mathbb{R}^{m \times n_{c}}$ define

$$
\delta_{A_{c}} \triangleq A_{c}^{\prime}-A_{c}, \quad \delta_{B_{c}} \triangleq B_{c}^{\prime}-B_{c}, \quad \delta_{C_{c}} \triangleq C_{c}^{\prime}-C_{c}
$$

and

$$
\left\|\left(\delta_{A_{c}}, \delta_{B_{c}}, \delta_{C_{c}}\right)\right\| \triangleq\left\|\delta_{A_{c}}\right\|+\left\|\delta_{B_{c}}\right\|+\left\|\delta_{C_{c}}\right\| .
$$

Furthermore, let $\tilde{A}^{\prime}, \tilde{V}^{\prime}$ and $\tilde{R}^{\prime}$ denote $\tilde{A}, \tilde{V}$ and $\tilde{R}$ with $\left(A_{c}, B_{c}, C_{c}\right)$ replaced by 
$\left(A_{c}^{\prime}, B_{c}^{\prime}, C_{c}^{\prime}\right)$ and define

$$
\begin{aligned}
\delta_{\tilde{A}} \triangleq \tilde{A}^{\prime}-\tilde{A} & =\left[\begin{array}{cc}
0 & B \delta_{C_{c}} \\
\delta_{B_{c}} C & \delta_{A_{c}}
\end{array}\right], \\
\delta_{\tilde{V}} \triangleq \tilde{V}^{\prime}-\tilde{V} & =\left[\begin{array}{cc}
0 & 0 \\
0 & B_{c} V_{2} \delta_{B_{c}}^{T}+\delta_{B_{c}} V_{2} B_{c}^{T}+\delta_{B_{c}} V_{2} \delta_{B_{c}}^{T}
\end{array}\right], \\
\delta_{\tilde{R}} \triangleq \tilde{R}^{\prime}-\tilde{R} & =\left[\begin{array}{cc}
0 & 0 \\
0 & C_{c}^{T} R_{2} \delta_{C_{c}}+\delta_{C_{c}}^{T} R_{2} C_{c}+\delta_{C_{c}}^{T} R_{2} \delta_{C_{c}}
\end{array}\right] .
\end{aligned}
$$

We shall also write $\tilde{Q}^{\prime}, \tilde{P}^{\prime}$ for $\tilde{Q}, \tilde{P}$ as given by (4.3) and (4.5) with $\tilde{A}, \tilde{V}, \tilde{R}$ replaced by $\tilde{A}^{\prime}, \tilde{V}^{\prime}, \tilde{R}^{\prime}$ and define

$$
\delta_{\tilde{Q}} \triangleq \tilde{Q}^{\prime}-\tilde{Q}, \quad \delta_{\tilde{P}} \triangleq \tilde{P}^{\prime}-\tilde{P} .
$$

Lemma 4.5. $\mathscr{A}$ is open.

Proof. Let $\left(A_{c}, B_{c}, C_{c}\right) \in \mathscr{A}$ be arbitrary and consider the open set

$$
N \triangleq\left\{\left(A_{c}^{\prime}, B_{c}^{\prime}, C_{c}^{\prime}\right) \in \mathbb{R}^{n_{c} \times n_{c}} \times \mathbb{R}^{n_{c} \times l} \times \mathbb{R}^{m \times n_{c}}:\left\|\left(\delta_{A_{c}}, \delta_{B_{c}}, \delta_{C_{c}}\right)\right\|<\beta / 2 \alpha \gamma\right\},
$$

where $\gamma \triangleq \max \{1,\|B\|,\|C\|\}$. Then, since $\tilde{A}^{\prime}=\tilde{A}+\delta_{\tilde{A}}$ and $\delta_{\tilde{A}} \in \mathscr{B}(\tilde{\mathscr{H}})$ it follows from Theorem 2.1, p. 497 of [50], that for all $\left(A_{c}^{\prime}, B_{c}^{\prime}, C_{c}^{\prime}\right) \in N$ and $t \geqq 0$,

$$
\left\|e^{\tilde{A}^{\prime} t}\right\| \leqq \alpha e^{\left(-\beta+\alpha\left\|\delta_{\tilde{A}}\right\|\right) t} \leqq \alpha e^{-\beta t / 2} .
$$

Hence, $N \subset \mathscr{A}$, as desired.

LEMMA 4.6. There exists $c>0$ such that

$$
\begin{gathered}
\left\|\delta_{\tilde{Q}}\right\| \leqq c\left\|\left(\delta_{A_{c}}, \delta_{B_{c}}, \delta_{C_{c}}\right)\right\|, \\
\left\|\delta_{\tilde{P}}\right\| \leqq c\left\|\left(\delta_{A_{c}}, \delta_{B_{c}}, \delta_{C_{c}}\right)\right\|,
\end{gathered}
$$

for all $\left(A_{c}^{\prime}, B_{c}^{\prime}, C_{c}^{\prime}\right) \in N$, where $N \subset \mathscr{A}$ is the open neighborhood of $\left(A_{c}, B_{c}, C_{c}\right)$ defined by (4.14).

Proof. We consider (4.15) only. Since $\left\|e^{\tilde{A}^{\prime} t}\right\| \leqq \alpha e^{-\beta t / 2}, t \geqq 0,\left(A_{c}^{\prime}, B_{c}^{\prime}, C_{c}^{\prime}\right) \in N$, it follows that

$$
\begin{aligned}
\left\|\delta_{\tilde{Q}}\right\| \leqq & \int_{0}^{\infty}\left\|e^{\tilde{A}^{\prime} t} \tilde{V}^{\prime} e^{\tilde{A}^{\prime *} t}-e^{\tilde{A} t} \tilde{V} e^{\tilde{A}^{*} t}\right\| d t \\
\leqq & \int_{0}^{\infty}\left\{\left\|e^{\tilde{A}^{\prime} t}\right\|\left\|\tilde{V}^{\prime}\right\|\left\|e^{\tilde{A}^{\prime *} t}-e^{\tilde{A}^{*} t}\right\|+\left\|e^{\tilde{A^{\prime} t}}\right\|\left\|\delta_{\tilde{V}}\right\|\left\|e^{\tilde{A}^{*} t}\right\|+\left\|e^{\tilde{A}^{\prime} t}-e^{\tilde{A} t}\right\|\|\tilde{V}\|\left\|e^{\tilde{A}^{*} t}\right\|\right\} d t \\
& +\alpha^{2}\left\|\delta_{\tilde{V}}\right\| \int_{0}^{\infty} e^{-3 \beta t / 2} d t+\alpha\|\tilde{V}\| \int_{0}^{\infty}\left\|e^{\left(\tilde{A}+\tilde{\delta}_{\tilde{A}}\right) t}-e^{\tilde{A} t}\right\| e^{-\beta t / 2} d t \\
= & \alpha\left(2\|\tilde{V}\|+\left\|\delta_{\tilde{V}}\right\|\right) \int_{0}^{\infty}\left\|e^{\left(\tilde{A}+\delta_{\tilde{A}}\right) t}-e^{\tilde{A} t}\right\| e^{-\beta t / 2} d t+\frac{2 \alpha^{2}}{3 \beta}\left\|\delta_{\tilde{V}}\right\| .
\end{aligned}
$$

From [50, p. 497], it follows that the perturbed semigroup $e^{\left(\tilde{A}+\delta_{\tilde{A}}\right) t}$ has an expansion

$$
e^{\left(\tilde{A}+\delta_{\tilde{A}}\right) t}=e^{\tilde{A} t}+\sum_{i=1}^{\infty} U_{i}(t), \quad t \geqq 0,
$$


where $U_{i}(t) \in \mathscr{B}(\tilde{\mathscr{C}}), t \geqq 0$, satisfy the estimates

$$
\left\|U_{i}(t)\right\| \leqq \alpha^{i+1}\left\|\delta_{\tilde{A}}\right\|^{i} e^{-\beta t} t^{i} / i !
$$

Hence, for all $\left(A_{c}^{\prime}, B_{c}^{\prime}, C_{c}^{\prime}\right) \in N$,

$$
\left\|e^{\left(\tilde{A}+\delta_{\tilde{A}}\right) t}-e^{\tilde{A} t}\right\| \leqq \sum_{i=1}^{\infty}\left\|U_{i}(t)\right\| \leqq \alpha e^{-\beta t}\left[e^{\alpha\left\|\delta_{\tilde{A}}\right\| t}-1\right] .
$$

From (4.17), (4.18) and the relations $\left\|\delta_{\tilde{A}}\right\| \leqq \gamma\left\|\left(\delta_{A_{c}}, \delta_{B_{c}}, \delta_{C_{c}}\right)\right\|<\beta / 2 \alpha$ and

$$
\int_{0}^{\infty}\left[e^{\alpha \| \delta_{\tilde{A}}^{\| t}}-1\right] e^{-3 \beta t / 2} d t<\frac{\alpha \gamma}{3 \beta^{2}}\left\|\left(\delta_{A_{c}}, \delta_{B_{c}}, \delta_{C_{c}}\right)\right\|
$$

it follows that

$$
\begin{aligned}
\left\|\delta_{\tilde{Q}}\right\| \leqq & \frac{2 \alpha^{3} \gamma}{3 \beta^{2}}\left(2\|\tilde{V}\|+\left\|\delta_{\tilde{V}}\right\|\right)\left\|\left(\delta_{A_{c}}, \delta_{B_{c}}, \delta_{C_{c}}\right)\right\| \\
& +\frac{2 \alpha^{2}}{3 \beta}\left(2\left\|B_{c} V_{2}\right\|\left\|\delta_{B_{c}}\right\|+\left\|V_{2}\right\|\left\|\delta_{B_{c}}\right\|^{2}\right),
\end{aligned}
$$

which yields (4.15). $\quad \square$

Since $\tilde{Q}, \tilde{P} \in \mathscr{B}(\tilde{\mathscr{H}})$ we can write

$$
\tilde{Q}=\left[\begin{array}{ll}
Q_{1} & Q_{12} \\
Q_{12}^{*} & Q_{2}
\end{array}\right], \quad \tilde{P}=\left[\begin{array}{ll}
P_{1} & P_{12} \\
P_{12}^{*} & P_{2}
\end{array}\right],
$$

where $Q_{1} \in \mathscr{B}(\mathscr{H}), Q_{12} \in \mathscr{B}\left(\mathbb{R}^{n_{c}}, \mathscr{H}\right), Q_{2} \in \mathbb{R}^{n_{c} \times n_{c}}$ and similarly for $P_{1}, P_{12}$ and $P_{2}$. Note that $Q_{1}, Q_{2}, P_{1}$ and $P_{2}$ are nonnegative definite. Also, define the notation

$$
\tilde{P} \tilde{Q}=\left[\begin{array}{ll}
Z_{1} & Z_{12} \\
Z_{21} & Z_{2}
\end{array}\right],
$$

where

$$
\begin{array}{ll}
Z_{1} \triangleq P_{1} Q_{1}+P_{12} Q_{12}^{*}, & Z_{12} \triangleq P_{1} Q_{12}+P_{12} Q_{2}, \\
Z_{21} \triangleq P_{12}^{*} Q_{1}+P_{2} Q_{12}^{*}, & Z_{2} \triangleq P_{12}^{*} Q_{12}+P_{2} Q_{2},
\end{array}
$$

and, for $\left(A_{c}^{\prime}, B_{c}^{\prime}, C_{c}^{\prime}\right) \in \mathscr{A}$, let

$$
\delta_{J}\left(\delta_{A_{c}}, \delta_{B_{c}}, \delta_{C_{c}}\right) \triangleq J\left(A_{c}^{\prime}, B_{c}^{\prime}, C_{c}^{\prime}\right)-J\left(A_{c}, B_{c}, C_{c}\right) .
$$

LeMmA 4.7. Let $\left(A_{c}^{\prime}, B_{c}^{\prime}, C_{c}^{\prime}\right) \in \mathscr{A}$. Then

$$
\delta_{J}\left(\delta_{A_{c}}, \delta_{B_{c}}, \delta_{C_{c}}\right)=\mathscr{L}\left(\delta_{A_{c}}, \delta_{B_{c}}, \delta_{C_{c}}\right)+o\left(\left\|\left(\delta_{A_{c}}, \delta_{B_{c}}, \delta_{C_{c}}\right)\right\|\right),
$$

where

$$
\begin{aligned}
\mathscr{L}\left(\delta_{A_{c}}, \delta_{B_{c}}, \delta_{C_{c}}\right) \triangleq & 2 \operatorname{tr}\left[Z_{2} \delta_{A_{c}}\right]+2 \operatorname{tr}\left[\left(V_{2} B_{c}^{T} P_{2}+C Z_{21}^{*}\right) \delta_{B_{c}}\right] \\
& \left.+2 \operatorname{tr}\left[Q_{2} C_{c}^{T} R_{2}+Z_{12}^{*} B\right) \delta_{C_{c}}\right]
\end{aligned}
$$

and

$$
\lim _{\left(\delta_{A_{c}}, \delta_{B_{c}}, \delta_{C_{c}}\right) \rightarrow 0}\left\|\left(\delta_{A_{c}}, \delta_{B_{c}}, \delta_{C_{c}}\right)\right\|^{-1} o\left(\left\|\left(\delta_{A_{c}}, \delta_{B_{c}}, \delta_{C_{c}}\right)\right\|\right)=0 .
$$

Proof. Combining (4.8) and (4.10) with (4.6), $J$ can be written as

$$
J\left(A_{c}, B_{c}, C_{c}\right)=\operatorname{tr}[\tilde{Q} \tilde{R}+\tilde{P} \tilde{V}]+\frac{1}{2} \operatorname{tr}\left[\tilde{Q} \operatorname{cl}(\tilde{A} * \tilde{P}+\tilde{P} \tilde{A})+\tilde{P} \operatorname{cl}\left(\tilde{A} \tilde{Q}+\tilde{Q} \tilde{A}^{*}\right)\right],
$$


and likewise for $\left(A_{c}^{\prime}, B_{c}^{\prime}, C_{c}^{\prime}\right)$, where "cl" denotes closure (i.e., extension) of a bounded operator to all of $\tilde{\mathscr{H}}$. Now using the identity

$$
\operatorname{tr}\left[\tilde{Q}^{\prime} \tilde{R}^{\prime}+\tilde{P}^{\prime} \tilde{V}^{\prime}\right]-\operatorname{tr}[\tilde{Q} \tilde{R}+\tilde{P} \tilde{V}]=\operatorname{tr}\left[\tilde{Q} \delta_{\tilde{R}}+\tilde{P} \delta_{\tilde{V}}\right]+\operatorname{tr}\left[\delta_{\tilde{Q}} \tilde{R}^{\prime}+\delta_{\tilde{P}} \tilde{V}^{\prime}\right]
$$

we can compute

$$
\begin{aligned}
\delta_{J}\left(\delta_{A_{c}}, \delta_{B_{c}}, \delta_{C_{c}}\right)= & \operatorname{tr}\left[\tilde{Q} \delta_{\tilde{R}}+\tilde{P} \delta_{\tilde{V}}\right]+\frac{1}{2} \operatorname{tr}\left[\tilde{Q} \operatorname{cl}\left(\tilde{A}^{*}\left(\tilde{P}+\delta_{\tilde{P}}\right)+\left(\tilde{P}+\delta_{\tilde{P}}\right) \tilde{A}^{\prime}\right)\right] \\
& +\frac{1}{2} \operatorname{tr}\left[\delta_{\tilde{Q}} \operatorname{cl}\left(\tilde{A}^{\prime *} \tilde{P}^{\prime}+\tilde{P}^{\prime} \tilde{A}^{\prime}\right)\right] \\
& +\frac{1}{2} \operatorname{tr}\left[\tilde{P} \operatorname{cl}\left(\tilde{A}^{\prime}\left(\tilde{Q}+\delta_{\tilde{Q}}\right)+\left(\tilde{Q}+\delta_{\tilde{Q}}\right) \tilde{A}^{\prime *}\right)\right] \\
& +\frac{1}{2} \operatorname{tr}\left[\delta_{\tilde{P}} \operatorname{cl}\left(\tilde{A}^{\prime} \tilde{Q}^{\prime}+\tilde{Q}^{\prime} \tilde{A}^{\prime *}\right)\right] \\
& -\frac{1}{2} \operatorname{tr}\left[\tilde{Q} \operatorname{cl}\left(\tilde{A}^{*} \tilde{P}+\tilde{P} \tilde{A}\right)+\tilde{P} \operatorname{cl}\left(\tilde{A} \tilde{Q}+\tilde{Q} \tilde{A}^{*}\right)\right] \\
& +\operatorname{tr}\left[\delta_{\tilde{Q}} \tilde{R}^{\prime}+\delta_{\tilde{P}} \tilde{V}^{\prime}\right] .
\end{aligned}
$$

Using $\tilde{A}^{\prime}=\tilde{A}+\delta_{\tilde{A}}$ and combining the second, fourth and sixth terms yields

$$
\delta_{J}\left(\delta_{A_{c}}, \delta_{B_{c}}, \delta_{C_{c}}\right)=\Lambda+\Omega \text {, }
$$

where

$$
\begin{aligned}
& \Lambda \triangleq \\
& \quad \operatorname{tr}\left[\tilde{Q} \delta_{\tilde{R}}+\tilde{P} \delta_{\tilde{V}}\right]+\frac{1}{2} \operatorname{tr}\left[\tilde{Q}\left(\delta_{\tilde{A}}^{*} \tilde{P}+\tilde{P} \delta_{\tilde{A}}\right)+\tilde{P}\left(\delta_{\tilde{A}} \tilde{Q}+\tilde{Q} \delta_{\tilde{A}}^{*}\right)\right] \\
& \quad=\operatorname{tr}\left[\tilde{Q} \delta_{\tilde{R}}+\tilde{P} \delta_{\tilde{V}}\right]+2 \operatorname{tr}\left[\delta_{\tilde{A}} \tilde{Q} \tilde{P}\right]
\end{aligned}
$$

and

$$
\begin{aligned}
\Omega \triangleq & \frac{1}{2} \operatorname{tr}\left[\tilde{Q} \operatorname{cl}\left(\tilde{A}^{\prime *} \delta_{\tilde{P}}+\delta_{\tilde{P}} \tilde{A}^{\prime}\right)+\tilde{P} \operatorname{cl}\left(\tilde{A}^{\prime} \delta_{\tilde{Q}}+\delta_{\tilde{Q}} \tilde{A}^{\prime *}\right)\right] \\
& +\frac{1}{2} \operatorname{tr}\left[\delta_{\tilde{Q}} \operatorname{cl}\left(\tilde{A}^{\prime *} \tilde{P}^{\prime}+\tilde{P}^{\prime} \tilde{A}^{\prime}\right)+\delta_{\tilde{P}} \operatorname{cl}\left(\tilde{A}^{\prime} \tilde{Q}^{\prime}+\tilde{Q}^{\prime} \tilde{A}^{\prime *}\right)\right]+\operatorname{tr}\left[\delta_{\tilde{Q}} \tilde{R}^{\prime}+\delta_{\tilde{P}} \tilde{V}^{\prime}\right]
\end{aligned}
$$

Computing

$$
\operatorname{tr}\left[\tilde{Q} \delta_{\tilde{R}}+\tilde{P} \delta_{\tilde{V}}\right]=2 \operatorname{tr}\left[V_{2} B_{c}^{T} P_{2} \delta_{B_{c}}\right]+2 \operatorname{tr}\left[Q_{2} C_{c}^{T} R_{2} \delta_{C_{c}}\right]+\operatorname{tr}\left[P_{2} \delta_{B_{c}} V_{2} \delta_{B_{c}}^{T}+Q_{2} \delta_{C_{c}}^{T} R_{2} \delta_{C_{c}}\right]
$$

and

$$
2 \operatorname{tr}\left[\delta_{\tilde{A}} \tilde{Q} \tilde{P}\right]=2 \operatorname{tr}\left[Z_{2} \delta_{A_{c}}\right]+2 \operatorname{tr}\left[C Z_{21}^{*} \delta_{B_{c}}\right]+2 \operatorname{tr}\left[Z_{12}^{*} B \delta_{C_{c}}\right]
$$

and retaining first-order terms, we obtain (4.20).

To evaluate $\Omega$, use (4.8) and (4.10) to replace $\tilde{R}^{\prime}$ and $\tilde{V}^{\prime}$ in the last term in $\Omega$ and write $\tilde{A}^{\prime}=\tilde{A}+\delta_{\tilde{A}}$, to obtain

$$
\begin{aligned}
\Omega= & \frac{1}{2} \operatorname{tr}\left[\tilde{Q} \operatorname{cl}\left(\tilde{A}^{*} \delta_{\tilde{P}}+\delta_{\tilde{P}} \tilde{A}\right)+\tilde{P} \operatorname{cl}\left(\tilde{A} \delta_{\tilde{Q}}+\delta_{\tilde{Q}} \tilde{A}^{*}\right)\right] \\
& +\frac{1}{2} \operatorname{tr}\left[\tilde{Q}\left(\delta_{\tilde{A}}^{*} \delta_{\tilde{P}}+\delta_{\tilde{P}} \delta_{\tilde{A}}\right)+\tilde{P}\left(\delta_{\tilde{A}} \delta_{\tilde{Q}}+\delta_{\tilde{Q}} \delta_{\tilde{A}}^{*}\right)\right] \\
& -\frac{1}{2} \operatorname{tr}\left[\delta_{\tilde{Q}} \operatorname{cl}\left(\tilde{A}^{\prime *} \tilde{P}^{\prime}+\tilde{P}^{\prime} \tilde{A}^{\prime}\right)+\delta_{\tilde{P}} \operatorname{cl}\left(\tilde{A}^{\prime} \tilde{Q}^{\prime}+\tilde{Q}^{\prime} \tilde{A}^{\prime *}\right)\right]
\end{aligned}
$$

Next we note that

$$
\operatorname{tr}\left[\tilde{Q} \operatorname{cl}\left(\tilde{A}^{*} \delta_{\tilde{P}}+\delta_{\tilde{P}} \tilde{A}^{*}\right)\right]=\operatorname{tr}\left[\delta_{\tilde{P}} \operatorname{cl}\left(\tilde{A} \tilde{Q}+\tilde{Q} \tilde{A}^{*}\right)\right] .
$$

To see this we observe that by arguments similar to those used in the proof of Lemma 4.4 and the fact that $\delta_{\tilde{P}}: \mathscr{D}(\tilde{A}) \rightarrow \mathscr{D}\left(\tilde{A}^{*}\right)$ it follows that

$$
\delta_{\tilde{P}}=-\int_{0}^{\infty} e^{\tilde{A}^{*} t} \operatorname{cl}\left(\tilde{A}^{*} \delta_{\tilde{P}}+\delta_{\tilde{P}} \tilde{A}\right) e^{\tilde{A} t} d t .
$$

Now, using the technique of Lemma 4.3 with the role of $\tilde{R}$ played by $-\operatorname{cl}\left(\tilde{A}^{*} \delta_{\tilde{P}}+\delta_{\tilde{P}} \tilde{A}\right)$, 
we see that

$$
\operatorname{tr}\left[\tilde{Q} \operatorname{cl}\left(\tilde{A}^{*} \delta_{\tilde{P}}+\delta_{\tilde{P}} \tilde{A}\right)\right]=-\operatorname{tr}\left[\delta_{\tilde{P}} \tilde{V}\right]=\operatorname{tr}\left[\delta_{\tilde{P}} \operatorname{cl}\left(\tilde{A} \tilde{Q}+\tilde{Q} \tilde{A}^{*}\right)\right] .
$$

Similarly, it can be shown that

$$
\operatorname{tr}\left[\tilde{P} \operatorname{cl}\left(\tilde{A} \delta_{\tilde{Q}}+\delta_{\tilde{Q}} \tilde{A}^{*}\right)\right]=\operatorname{tr}\left[\delta_{\tilde{Q}} \operatorname{cl}\left(\tilde{A}^{*} \tilde{P}+\tilde{P} \tilde{A}\right)\right] .
$$

Now substitute (4.23) and (4.24) into (4.22) and rearrange the second term in (4.22) so that

$$
\begin{aligned}
\Omega= & \frac{1}{2} \operatorname{tr}\left[\delta_{\tilde{Q}} \operatorname{cl}\left(\tilde{A}^{*} \tilde{P}+\tilde{P} \tilde{A}\right)+\delta_{\tilde{P}} \operatorname{cl}\left(\tilde{A} \tilde{Q}+\tilde{Q} \tilde{A}^{*}\right)\right] \\
& +\frac{1}{2} \operatorname{tr}\left[\delta_{\tilde{Q}}\left(\delta_{\tilde{A}}^{*} \tilde{P}+\tilde{P} \delta_{\tilde{A}}\right)+\delta_{\tilde{P}}\left(\delta_{\tilde{A}} \tilde{Q}+\tilde{Q} \delta_{\tilde{A}}^{*}\right)\right] \\
& -\frac{1}{2} \operatorname{tr}\left[\delta_{\tilde{Q}} \operatorname{cl}\left(\tilde{A}^{\prime *} \tilde{P}^{\prime}+\tilde{P}^{\prime} \tilde{A}^{\prime}\right)+\delta_{\tilde{P}} \operatorname{cl}\left(\tilde{A}^{\prime} \tilde{Q}^{\prime}+\tilde{Q}^{\prime} \tilde{A}^{\prime *}\right)\right] \\
= & -\frac{1}{2} \operatorname{tr}\left[\delta_{\tilde{Q}} \operatorname{cl}\left(\tilde{A}^{\prime *} \delta_{\tilde{P}}+\delta_{\tilde{P}} \tilde{A}^{\prime}\right)+\delta_{\tilde{P}} \operatorname{cl}\left(\tilde{A}^{\prime} \delta_{\tilde{Q}}+\delta_{\tilde{Q}} \tilde{A}^{\prime *}\right)\right] .
\end{aligned}
$$

Using (4.8) to obtain

$$
0=\tilde{A}^{\prime} \delta_{\tilde{Q}}+\delta_{\tilde{Q}} \tilde{A}^{\prime *}+\delta_{\tilde{A}} \tilde{Q}+\tilde{Q} \delta_{\tilde{A}}^{*}+\delta_{\tilde{V}}
$$

and (4.10) to obtain a similar relation involving $\tilde{P}$, we have

$$
\left.\Omega=\operatorname{tr}\left[\delta_{\tilde{Q}}\left(\delta_{\tilde{A}}^{*} \tilde{P}+\tilde{P} \delta_{\tilde{A}}+\delta_{\tilde{R}}\right)\right]+\operatorname{tr}\left[\delta_{\tilde{P}} \tilde{Q}+\tilde{Q} \delta_{\tilde{A}}^{*}+\delta_{\tilde{V}}\right)\right] .
$$

Restricting $\left(A_{c}^{\prime}, B_{c}^{\prime}, C_{c}^{\prime}\right)$ to $N$ (see (4.14)), using Lemma 4.6 and noting that $\delta_{\tilde{A}}$ and $\delta_{\tilde{R}}$ have finite rank, it follows that there exists $c_{1}>0$ such that

$$
\|\Omega\| \leqq c_{1}\left\|\left(\delta_{A_{c}}, \delta_{B_{c}}, \delta_{C_{c}}\right)\right\|^{2} \text {. }
$$

Combining $\Omega$ with the second-order terms in $\Lambda$ yields the desired result.

LeMma 4.8. $\mathscr{A}_{+}$is open.

Proof. From the "generic" property of controllability and observability [62, p. 44] there exists an open neighborhood of $\left(A_{c}, B_{c}, C_{c}\right)$ each of whose elements is minimal. Combining this fact with Lemma 4.5 yields the desired result.

LEMMA 4.9. $Q_{2}$ and $P_{2}$ are positive definite.

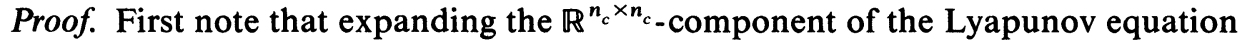
(4.8) yields (4.50) below. By a minor extension of results from [66] or [67], (4.50) can be rewritten as

$$
0=\left(A_{c}+B_{c} C Q_{12} Q_{2}^{+}\right) Q_{2}+Q_{2}\left(A_{c}+B_{c} C Q_{12} Q_{2}^{+}\right)^{T}+B_{c} V_{2} B_{c}^{T},
$$

where $Q_{2}^{+}$is the Moore-Penrose or Drazin generalized inverse of $Q_{2}$. Next note that since $\left(A_{c}, B_{c}\right)$ is controllable then so is $\left(A_{c}+B_{c} C Q_{12} Q_{2}^{+}, B_{c} V_{2}^{1 / 2}\right)$. Now, since $Q_{2}$ and $B_{c} V_{2} B_{c}^{T}$ are nonnegative definite, it follows from [62, Lemma 12.2] that $Q_{2}$ is positive definite. Similar arguments show that $P_{2}$ is positive definite.

Having established Lemmas 4.1-4.9, we can now proceed with the proof of the Main Theorem. Let $\left(A_{c}, B_{c}, C_{c}\right) \in \mathscr{A}_{+}$be as in the Main Theorem and consider (4.19) with $\left(A_{c}^{\prime}, B_{c}^{\prime}, C_{c}^{\prime}\right)$ confined to $\mathscr{A}_{+}$. Because $\mathscr{L}: \mathbb{R}^{n_{c} \times n_{c}} \times \mathbb{R}^{n_{c} \times l} \times \mathbb{R}^{m \times n_{c}} \rightarrow \mathbb{R}$ is a bounded linear functional and $\mathscr{A}_{+}$is open, the convergence in (4.21) implies that $\mathscr{L}$ is precisely the Frechet derivative of $J$ with respect to $\left(A_{c}, B_{c}, C_{c}\right)$. Since $\mathscr{A}_{+}$is open, the optimality of $\left(A_{c}, B_{c}, C_{c}\right)$ implies

$$
\mathscr{L}\left(\delta_{A_{c}}, \delta_{B_{c}}, \delta_{C_{c}}\right)=0
$$

for all $\left(\delta_{A_{c}}, \delta_{B_{c}}, \delta_{C_{c}}\right)$. Clearly, $(4.26)$ is equivalent to

$$
Z_{2}=0 \text {, }
$$




$$
\begin{aligned}
& V_{2} B_{c}^{T} P_{2}+C Z_{21}^{*}=0, \\
& Q_{2} C_{c}^{T} R_{2}+Z_{12}^{*} B=0 .
\end{aligned}
$$

Thus, $B_{c}$ and $C_{c}$ are given by

$$
\begin{aligned}
& B_{c}=-P_{2}^{-1} Z_{21} C^{*} V_{2}^{-1}, \\
& C_{c}=-R_{2}^{-1} B^{*} Z_{12} Q_{2}^{-1} .
\end{aligned}
$$

Although $B_{c}$ and $C_{c}$ are now determined in terms of $\tilde{Q}$ and $\tilde{P}, A_{c}$ remains to be found. Moreover, $\tilde{Q}$ and $\tilde{P}$ themselves depend (via (4.8) and (4.10)) on $B_{c}$ and $C_{c}$. Hence our task now is to consolidate and simplify (4.7)-(4.10), (4.27), (4.30) and (4.31) to obtain the more tractable conditions (3.9)-(3.18). To this end let us define new variables
$(4.32 \mathrm{a}, \mathrm{b})$
$Q \triangleq Q_{1}-Q_{12} Q_{2}^{-1} Q_{12}^{*}$
$P \triangleq P_{1}-P_{12} P_{2}^{-1} P_{12}^{*}$
$(4.33 \mathrm{a}, \mathrm{b})$
$\hat{Q} \triangleq Q_{12} Q_{2}^{-1} Q_{12}^{*}$,
$\hat{P} \triangleq P_{12} P_{2}^{-1} P_{12}^{*}$.

Clearly, $\hat{Q}$ and $\hat{P}$ are nonnegative definite and have finite rank. Since by Lemma 4.2 $\tilde{Q}, \tilde{P} \in \mathscr{B}_{1}(\tilde{\mathscr{H}})$, it can be seen that $Q_{1}, P_{1} \in \mathscr{B}_{1}(\mathscr{H})$, which implies $Q, P \in \mathscr{B}_{1}(\mathscr{H})$. To show that $Q$ and $P$ are nonnegative definite, note that $Q$ is the $\mathscr{B}(\mathscr{H})$-component of the nonnegative-definite operator $2 \tilde{Q} 2^{*} \in \mathscr{B}(\tilde{\mathscr{H}})$, where

$$
2 \triangleq\left[\begin{array}{cc}
I_{\mathscr{K}} & -Q_{12} Q_{2}^{-1} \\
0 & -I_{n_{c}}
\end{array}\right] \text {. }
$$

Similarly, $P$ is nonnegative definite.

From the domain conditions (4.7) and (4.9) it follows that
$(4.34 a, b)$
$Q_{1}: \mathscr{D}\left(A^{*}\right) \rightarrow \mathscr{D}(A)$,
$P_{1}: \mathscr{D}(A) \rightarrow \mathscr{D}\left(A^{*}\right)$,
$(4.35 \mathrm{a}, \mathrm{b})$

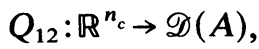
$P_{12}: \mathbb{R}^{n_{c}} \rightarrow \mathscr{D}\left(A^{*}\right)$,

which lead to (3.12) and (3.13).

Next note that (4.27) is equivalent to (3.8) with

$$
G \triangleq Q_{2}^{-1} Q_{12}^{*}, \quad \Gamma \triangleq-P_{2}^{-1} P_{12}^{*}
$$

and that (3.7) holds with

$$
M \triangleq Q_{2} P_{2} \text {. }
$$

Since $Q_{2}$ and $P_{2}$ are positive definite, Lemma 2.6 implies that $M$ is positive semisimple. We can also define $\tau=G^{*} \Gamma$ which, by (3.8) satisfies $\tau^{2}=\tau$. It is helpful to note the identities

$$
\begin{array}{ll}
\text { (4.38a, b) } & \hat{Q}=Q_{12} G=G^{*} Q_{12}^{*}, \quad \hat{P}=-P_{12} \Gamma=-\Gamma^{*} P_{12}^{*}, \\
(4.39 \mathrm{a}, \mathrm{b}) & \hat{Q}=G^{*} Q_{2} G, \quad \hat{P}=\Gamma^{*} P_{2} \Gamma, \\
(4.40 \mathrm{a}, \mathrm{b}) & \tau G^{*}=G^{*}, \quad \Gamma \tau=\Gamma, \\
(4.41 \mathrm{a}, \mathrm{b}) & \hat{Q}=\tau \hat{Q}, \quad \hat{P}=\hat{P} \tau, \\
(4.42) & \hat{Q} \hat{P}=-Q_{12} P_{12}^{*} .
\end{array}
$$

From (3.8) and (2.1) it follows that

$$
\begin{aligned}
& \rho(G)=\rho(\Gamma)=n_{c}, \\
& \rho\left(Q_{12}\right)=\rho\left(P_{12}\right)=n_{c} .
\end{aligned}
$$


Hence, (2.2) and (4.38) imply $n_{c}=\rho\left(Q_{12}\right)+\rho(G)-n_{c} \leqq \rho(\hat{Q}) \leqq \rho\left(Q_{12}\right)=n_{c}$, which yields (3.14a). Similarly, (3.14b) holds and (3.14c) follows from (2.2) and (4.42).

Using (4.38) and (4.39), the components of $\tilde{Q}$ and $\tilde{P}$ can be written in terms of $G, \Gamma, Q, P, \hat{Q}$ and $\hat{P}$ as

$$
\begin{array}{ll}
Q_{1}=Q+\hat{Q}, & P_{1}=P+\hat{P}, \\
Q_{12}=\hat{Q} \Gamma^{*}, & P_{12}=-\hat{P} G^{*}, \\
Q_{2}=\Gamma \hat{Q} \Gamma^{*}, & P_{2}=G \hat{P} G^{*} .
\end{array}
$$

Now (3.10) and (3.11) can be obtained by substituting (4.45)-(4.47) into (4.30) and (4.31).

Expanding the $\mathscr{B}(\mathscr{H}), \mathscr{B}\left(\mathbb{R}^{n_{c}}, \mathscr{H}\right)$ and $\mathbb{R}^{n_{c} \times n_{c}}$ components of $(4.8)$ and (4.10) yields

$$
\begin{aligned}
& 0=A Q_{1}+Q_{1} A^{*}+B C_{c} Q_{12}^{*}+Q_{12}\left(B C_{c}\right)^{*}+V_{1}, \\
& 0=A Q_{12}+Q_{12} A_{c}^{T}+B C_{c} Q_{2}+Q_{1}\left(B_{c} C\right)^{*}, \\
& 0=A_{c} Q_{2}+Q_{2} A_{c}^{T}+B_{c} C Q_{12}+Q_{12}^{*}\left(B_{c} C\right)^{*}+B_{c} V_{2} B_{c}^{T}, \\
& 0=A^{*} P_{1}+P_{1} A+\left(B_{c} C\right)^{*} P_{12}^{*}+P_{12} B_{c} C+R_{1}, \\
& 0=P_{12} A_{c}+A^{*} P_{12}+\left(B_{c} C\right)^{*} P_{2}+P_{1} B C_{c}, \\
& 0=A_{c}^{T} P_{2}+P_{2} A_{c}+\left(B C_{c}\right)^{*} P_{12}+P_{12}^{*} B C_{c}+C_{c}^{T} R_{2} C_{c} .
\end{aligned}
$$

Substituting (4.45)-(4.47) into (4.48)-(4.53), using the identities

and defining

$$
\begin{aligned}
& B_{c} C=\Gamma Q \bar{\Sigma}, \quad B C_{c}=-\Sigma P G^{*}, \\
& B_{c} V_{2} B_{c}^{T}=\Gamma Q \bar{\Sigma} Q \Gamma^{*}, \quad C_{c}^{T} R_{2} C_{c}=G P \Sigma P G^{*},
\end{aligned}
$$

we obtain

$$
A_{Q} \triangleq A-Q \bar{\Sigma}, \quad A_{P} \triangleq A-\Sigma P,
$$

$$
\begin{aligned}
& 0=A Q+Q A^{*}+A_{P} \hat{Q}+\hat{Q} A_{P}+V_{1}, \\
& 0=\left[A_{P} \hat{Q}+Q \bar{\Sigma} Q+\hat{Q}\left(\Gamma^{*} A_{c}^{T} G+\bar{\Sigma} Q\right)\right] \Gamma^{*}, \\
& 0=\Gamma\left[G^{*} A_{c} \Gamma \hat{Q}+Q \bar{\Sigma} \hat{Q}+Q \bar{\Sigma} Q+\hat{Q}\left(\Gamma^{*} A_{c}^{T} G+\bar{\Sigma} Q\right)\right] \Gamma^{*}, \\
& 0=A^{*} P+P A+A_{Q}^{*} \hat{P}+\hat{P} A_{Q}+R_{1}, \\
& 0=-\left[A_{Q}^{*} \hat{P}+P \Sigma P+\hat{P}\left(G^{*} A_{c} \Gamma+\Sigma P\right)\right] G^{*}, \\
& 0=G\left[\Gamma^{*} A_{c}^{T} G \hat{P}+P \Sigma \hat{P}+P \Sigma P+\hat{P}\left(G^{*} A_{c} \Gamma+\Sigma P\right)\right] G^{*} .
\end{aligned}
$$

We are now in a position to determine $A_{c}$ by computing (4.56) $-\Gamma(4.55)$ which yields (3.9). Alternatively, $A_{c}$ can be obtained by computing (4.59) $+G(4.58)$. As mentioned in $\S 3,(3.9)$ is valid since $G^{*}: \mathbb{R}^{n_{c}} \rightarrow \mathscr{D}(A)$ and $A_{c}^{T}$ is given by (3.26).

Next we substitute the expressions for $A_{c}$ and $A_{c}^{T}$ into (4.55), (4.56), (4.58) and (4.59) and compute the relations (4.55) $G, G^{*}(4.56) G,-(4.58) \Gamma$ and $\Gamma^{*}(4.59) \Gamma$ to obtain, respectively,

$$
\begin{aligned}
& 0=\left[A_{P} \hat{Q}+\hat{Q} A_{P}^{*}+Q \bar{\Sigma} Q\right] \tau^{*}, \\
& 0=\tau\left[A_{P} \hat{Q}+\hat{Q} A_{P}^{*}+Q \bar{\Sigma} Q\right] \tau^{*}, \\
& 0=\left[A_{Q}^{*} \hat{P}+\hat{P} A_{Q}+P \Sigma P\right] \tau, \\
& 0=\tau^{*}\left[A_{Q}^{*} \hat{P}+\hat{P} A_{Q}+P \Sigma P\right] \tau .
\end{aligned}
$$


Note that (4.60)-(4.63) are equivalent to (4.55), (4.56), (4.58) and (4.59) since $G$ and $\Gamma$ have full rank. Since $(4.61)=\tau(4.60)$ and $(4.63)=\tau^{*}(4.62),(4.61)$ and $(4.63)$ are superfluous and can be omitted. Thus we have derived (3.17) and (3.18).

To obtain (3.15) and (3.16) we need only compute the relations $(4.54)+\tau(4.60)-$ $(4.60)-(4.60)^{*}$ and $(4.57)+\tau^{*}(4.62)-(4.62)-(4.62)^{*}$ and use $(4.41)$.

Finally, to show that the preceding development entails no loss of generality in the optimality conditions we now use (3.9)-(3.18) to obtain (4.7)-(4.10) and (4.27)(4.29). Let $A_{c}, B_{c}, C_{c}, G, \Gamma, \tau, Q, P, \hat{Q}, \hat{P}$ be as in the theorem statement and define $Q_{1}, Q_{12}, Q_{2}, P_{1}, P_{12}, P_{2}$ by (4.45)-(4.47). Note that (3.12) and (3.13) imply (4.34) and (4.35) and hence (4.7) and (4.9). Using (3.8), (3.10), (3.11) and (3.22) it is easy to verify (4.27)-(4.29). Finally, substitute (4.32), (4.33) and (4.36) into (3.15)-(3.18), reverse the steps taken earlier in the proof and use (3.9)-(3.11) to obtain (4.8) and (4.10), which completes the proof.

5. Concluding remarks. This paper has considered the problem of quadratically optimal, steady-state, fixed-order dynamic compensation for linear infinite-dimensional systems. The Main Theorem presents the stationarity conditions of the optimization problem in a highly simplified and rigorous form. The "optimal projection equations" (3.15)-(3.18) (or, equivalently, (3.27)-(3.30)) of the Main Theorem reveal the essential structure of the first-order necessary conditions and display the central role played by the optimal projection $\tau$. The relationship of the Main Theorem to the standard finite-dimensional steady-state $L Q G$ problem can be demonstrated by replacing $\tau$ with the identity matrix and noting that (3.27) and (3.28) reduce immediately to the familiar pair of operator Riccati equations and that (3.29) and (3.30) yield the controllability and observability gramians of the controller.

Inasmuch as the Main Theorem is a fundamental generalization of classical steady-state $L Q G$ theory, a number of issues must be reexamined. Hence, in conclusion we should like to point out some possible extensions of the Main Theorem along with directions for further research.

1. Sufficiency theory. Although sufficient conditions for the existence of an optimal compensator were not investigated in this paper, auxiliary conditions based upon the structure of (3.15)-(3.18) could perhaps be imposed upon $Q, P, \hat{Q}$ and $\hat{P}$ to single out the global optimum from amongst the local minima. This would be similar to the situation in $L Q G$ theory where, under stabilizability and detectability hypotheses, optimal stabilizing $Q$ and $P$ are identified as the unique nonnegative-definite solutions of the pair of algebraic Riccati equations.

2. Stabilizability. Just as in the full-order $L Q G$ problem, one would expect a natural relationship between the structure of the optimal solution and stabilizability/detectability hypotheses. The results of [41], [42] and [68] could serve as a starting point in this regard.

3. Numerical algorithms. In practical situations, the distributed parameter system would be replaced by a high-order discretized model for which the matrix version (rather than the operator version) of the optimal projection equations could be solved numerically. A numerical algorithm for solving the matrix version of the optimal projection equations has been developed in [32] and [34]. The proposed computational scheme is fundamentally quite different from gradient search algorithms [17], [18], [21], [22], [24], [25], [28], [30] in that it operates through direct solution of the optimal projection equations by iterative refinement of the optimal projection.

4. Convergence. One of the principal uses for the optimal projection equations will be to understand the relationship between fixed-order dynamic-compensator 
designs which are optimal with respect to approximate models and the optimal fixed-order dynamic compensator for the distributed parameter system itself. By considering a sequence of $n$ th-order approximate models which converge to the distributed parameter system, conditions would be sought guaranteeing that the sequence of fixed-order compensators based on each approximate model approach the optimal dynamic compensator based upon the distributed parameter system (see [38]-[40]). This approach is analogous to the convergence results obtained in [7], [8] with the major difference being that the optimal projection equations permit the order of the compensator to remain fixed in accordance with real-world implementation constraints whereas in [7]-[9] the order of the compensator increases without bound.

5. Unbounded control and observation. An important generalization of the problem considered in this paper involves the case in which the input and output operators $B$ and $C$ are unbounded. The mathematical details for this problem are considerably more complex (see, e.g., [69]).

6. Singular observation noise/singular control weighting. As pointed out in [22], [33], [36] the assumptions of nonsingular control weighting and nonsingular observation noise preclude the use of direct output feedback as in

$$
u(t)=C_{c} x_{c}(t)+D_{c} y(t)
$$

since $J$ is undefined unless

$$
\operatorname{tr}\left[D_{c}^{T} R_{2} D_{c} V_{2}\right]=0\left(\Leftrightarrow R_{2} D_{c} V_{2}=0\right) .
$$

Although with due attention to (5.1) direct output feedback can be used in the singular case, the nature of the problem forebodes all of the difficulties associated with the singular $L Q G$ problem. Note that the deterministic output feedback problem [70], when viewed in this context, is highly singular.

7. Discrete-time system/discrete-time compensator. Digital implementation can be modelled by a discrete-time compensator with control of a continuous-time system facilitated by sampling and reconstruction devices. See [71], [73] for results in this direction.

8. Cross weighting/correlated disturbance and observation noise. This extension is straightforward and entirely analogous to the $L Q G$ case (see, e.g., [18, p. 351]).

Acknowledgments. We wish to thank Ardeth P. Grant for excellent and careful typing of the original manuscript version of this paper. Word processing and revision support was provided by Harris Corporation, GASD.

\section{REFERENCES}

[1] M. J. BALAS, Toward a more practical control theory for distributed parameter systems, in Control and Dynamic Systems, C. T. Leondes, ed., Advances in Theory and Applications, 19, Academic Press, New York, 1982.

[2] M. ATHANS, Toward a practical theory of distributed parameter systems, IEEE Trans. Automat. Control., AC-15 (1970), pp. 245-247.

[3] S. A. REIBLE, Acoustoelectric convolver technology for-spread-spectrum communications, IEEE Trans. Microwave Theory Tech., MTT-29 (1981), pp. 463-473.

[4] R. F. Curtain And A. J. Pritchard, Infinite Dimensional Linear Systems Theory, Springer-Verlag, New York, 1978.

[5] J. S. GiBSON, The Riccati integral equations for optimal control problems on Hilbert spaces, this Journal, 17 (1979), pp. 537-565. 
[6] A. V. Balakrishnan, Applied Functional Analysis, Springer-Verlag, New York, 1981.

[7] J. S. GiBSON, An analysis of optimal modal regulation: convergence and stability, this Journal, 19 (1981), pp. 686-707.

[8] - Linear-quadratic optimal control of hereditary differential systems: infinite dimensional Riccati equations and numerical approximations, this Journal, 21 (1983), pp. 95-139.

[8a] H. T. BANKS AND K. KUNISCH, The linear regulator problem for parabolic systems, this Journal, 22 (1984), pp. 684-698.

[9] H. T. BANKS, I. G. RosEn AND K. ITO, A spline based technique for computing Riccati operators and feedback controls in regulator problems for delay equations, SIAM J. Sci. Stat. Comput., 5 (1984), pp. 830-855.

[10] M. AOKI, Control of large-scale dynamic systems by aggregation, IEEE Trans. Automat. Control, AC-13 (1968), pp. 246-253.

[11] D. A. WILSON, Optimum solution of model-reduction problems, Proc. IEE, 117 (1970), pp. 1161-1165.

[12] B. C. MOORE, Principal component analysis in linear systems: controllability, observability, and model reduction, IEEE Trans. Automat. Control, AC-26 (1981), pp. 17-32.

[13] R. E. Skelton AND A. Yousuff, Component cost analysis of large scale systems, in Control and Dynamic Systems, C. T. Leondes, ed., Academic Press, New York, 1982.

[14] E. A. Jonckheere ANd L. M. Silverman, A new set of invariants for linear systems-application to reduced-order compensator design, IEEE Trans. Automat. Control, AC-28 (1983), pp. 953-964.

[15] A. Yousuff AND R. E. SKelton, Controller reduction by component cost analysis, IEEE Trans. Automat. Control, AC-29 (1984), pp. 520-530.

[16] T. L. Johnson AND M. AthANS, On the design of optimal constrained dynamic compensators for linear constant systems, IEEE Trans. Automat. Control, AC-15 (1970), pp. 658-660.

[17] W. S. Levine, T. L. Johnson AND M. Athans, Optimal limited state variable feedback controllers for linear systems, IEEE Trans. Automat. Control, AC-16 (1971), pp. 785-793.

[18] K. KwakernaAK And R. Sivan, Linear Optimal Control Systems, Wiley-Interscience, New York, 1972.

[19] D. B. ROM AND P. E. SARACHIK, The design of optimal compensators for linear constant systems with inaccessible states, IEEE Trans. Automat. Control, AC-18 (1973), pp. 509-512.

[20] M. SidAR AND B.-Z. KURTARAN, Optimal low-order controllers for linear stochastic systems, Int. J. Control, 22 (1975), pp. 377-387.

[21] J. M. MEndel ANd J. FeAther, On the design of optimal time-invariant compensators for linear stochastic time-invariant systems, IEEE Trans. Automat. Control, AC-20 (1975), pp. 653-657.

[22] S. BASUTHAKUR AND C. H. KNAPP, Optimal constant controllers for stochastic linear systems, IEEE Trans. Automat. Control, AC-20 (1975), pp. 664-666.

[23] R. B. ASHER AND J. C. DURRETT, Linear discrete stochastic control with a reduced-order dynamic compensator, IEEE Trans. Automat. Control, AC-21 (1976), pp. 626-627.

[24] W. J. NAEIJE AND O. H. BOSGRA, The design of dynamic compensators for linear multivariable systems, 1977 IFAC, Fredericton, New Brunswick, Canada, pp. 205-212.

[25] H. R. SIRISENA AND S. S. CHOI, Design of optimal constrained dynamic compensators for non-stationary linear stochastic systems, Int. J. Contr., 25 (1977), pp. 513-524.

[26] P. J. BlanVillain ANd T. L. JohnSON, Specific-optimal control with a dual minimal-order observerbased compensator, Int. J. Contr., 28 (1978), pp. 277-294.

[27] - Invariants of optimal minimal-order observer-based compensators, IEEE Trans. Automat. Control, AC-23 (1978), pp. 473-474.

[28] C. J. WENK AND C. H. KNAPP, Parameter optimization in linear systems with arbitrarily constrained controller structure, IEEE Trans. Automat. Control, AC-25 (1980), pp. 496-500.

[29] J. O'ReIlly, Optimal low-order feedback controllers for linear discrete-time systems, in Control and Dynamic Systems 16. C. T. Leondes, ed., Academic Press, New York, 1980.

[30] D. P. LOOZE AND N. R. SANDELL, JR., Gradient calculations for linear quadratic fixed control structure problems, IEEE Trans. Automat. Control, AC-25 (1980), pp. 285-288.

[31] D. C. HYLAND, Optimality conditions for fixed-order dynamic compensation of flexible spacecraft with uncertain parameters, AIAA 20th Aerospace Sciences Mtg., paper 82-0312, Orlando, FL, Jan. 1982.

[32] - The optimal projection approach to fixed-order compensation: Numerical methods and illustrative results, AIAA 21st Aerospace Sciences Mtg., paper 83-0303, Reno, NV, Jan. 1983.

[33] D. C. HYLAND AND D. S. BERNSTEIN, Explicit optimality conditions for fixed-order dynamic compensation, Proc. IEEE Conference on Decision and Control, San Antonio, TX, Dec. 1983, pp. 161-165.

[34] D. C. HYLAND, Comparison of various controller-reduction methods: Suboptimal versus optimal projection, Proc. AIAA Dynamics Specialists Conference, Palm Springs, CA, May 1984, pp. 382-389. 
[35] D. S. BERNSTEIN AND D. C. HYLAND, The optimal projection equations for fixed-order dynamic compensation of distributed parameter systems, Proc. AIAA Dynamics Specialists Conference, Palm Springs, CA, May 1984, pp. 396-400.

[36] - The optimal projection equations for fixed-order dynamic compensation, IEEE Trans. Automat. Control, AC-29 (1984), pp. 1034-1037.

[37] - The optimal projection approach to model reduction and the relationship between the methods of Wilson and Moore, Proc. IEEE Conference on Decision and Control, Las Vegas, NV, Dec. 1984, pp. $120-126$.

[37a] - The optimal projection equations for model reduction and the relationships among the methods of Wilson, Skelton and Moore, to appear.

[38] T. L. Johnson, Optimization of low order compensators for infinite dimensional systems, Proc. 9th IFIP Symposium on Optimization Techniques, Warsaw, Poland, September 1979, pp. 394-401.

[39] R. K. PEARSON, Optimal fixed-form compensators for large space structures, in ACOSS SIX (Active Control of Space Structures), RADC-TR-81-289, Final Technical Report, Rome Air Development Center, Griffiss AFB, New York, 1981.

[40] — Optimal velocity feedback control of flexible structures, Ph.D. dissertation, Dept. Electrical Engineering and Computer Science, Massachusetts Institute of Technology, Cambridge, 1982.

[41] R. F. Curtain, Compensators for infinite-dimensional linear systems, J. Franklin Inst., 315 (1983), pp. 331-346.

[42] J. M. SChUmACHER, A direct approach to compensator design for distributed parameter systems, this Journal, 21 (1983), pp. 823-836.

[43] D. L: RusSELL, Linear stabilization of the linear oscillator in Hilbert space, J. Math. Anal. Appl., 25 (1969), pp. 663-675.

[44] - Decay rates for weakly damped systems in Hilbert space obtained with control theoretic methods, J. Differential Equations, 19 (1975), pp. 344-370.

[45] M. J. BALAS, Modal control of certain flexible dynamic systems, this Journal, 16 (1978), pp. 450-462.

[46] - Feedback control of flexible systems, IEEE Trans. Automat. Control, AC-23 (1978), pp. 673-679.

[47] J. S. GiBSON, A note on stabilization of infinite dimensional linear oscillators by compact feedback, this Journal, 18 (1980), pp. 311-316.

[48] M. J. BALAS, Trends in large space structure control theory: Fondest hopes, wildest dreams, IEEE Trans. Automat. Control, AC-24 (1982), pp. 522-535.

[49] T. L. JOHNSON, Progress in modelling and control of flexible spacecraft, J. Franklin Inst., 315 (1983), pp. 495-520.

[49a] M. J. BALAS, Feedback control of dissipative hyperbolic distributed parameter systems with finite dimensional controllers, J. Math. Anal. Appl., 98 (1984), pp. 1-24.

[50] T. Kato, Perturbation Theory for Linear Operators, Springer-Verlag, New York, 1966.

[51] J. R. Ringrose, Compact Non-Self-Adjoint Operators, Van Nostrand Reinhold, London, 1971.

[52] I. C. Gohberg AND M. G. KREIN, Introduction to the Theory of Linear Nonselfadjoint Operators, Translations of Mathematical Monographs, Vol. 18, American Mathematical Society, Providence, RI, 1966.

[52a] M. S. BRoDSkiI, Triangular and Jordan Representations of Linear Operators, Translations of Mathematical Monographs, Vol. 32, American Mathematical Society, Providence, RI, 1971.

[53] I. Gohberg ANd S. GoldberG, Basic Operator Theory, Birkhauser, Boston, 1981.

[54] F. R. Gantmacher, The Theory of Matrices, Vol. I, Chelsea, New York, 1977.

[55] C. R. RAo AND S. K. Mitra, Generalized Inverse of Matrices and Its Applications, John Wiley, New York, 1971.

[56] B. Noble and J. W. Daniel, Applied Linear Algebra, Second edition, Prentice-Hall, Englewood Cliffs, NJ, 1977.

[57] R. F. Curtain And A. J. Pritchard, Functional Analysis in Modern Applied Mathematics, Academic Press, London, 1977.

[58] S. Chakrabarti, B. B. Battacharyya And M. N. S. Swamy, On simultaneous diagonalization of a collection of hermitian matrices, Matrix and Tensor Quart., 29 (1978), pp. 35-54.

[59] C. T. MUllis AND R. A. ROBERTS, Synthesis of minimum roundoff noise fixed point digital filters, IEEE Trans. Circ. Syst., CAS-23 (1976), pp. 551-562.

[60] A. J. LAUB, Computation of balancing transformation, Proc. 1980 Joint Automation Control Conference, San Francisco, CA, Aug. 1980.

[61] E. JONCKHEERE, Open-loop and closed loop approximations of linear systems and associated balanced realizations, 1982 Symposium on Circuits and Systems, Rome, May 1982.

[62] W. M. Wonham, Linear Multivariable Control: A Geometric Approach, Springer-Verlag, New York, 1974. 
[63] D. C. LAY, Spectral properties of generalized inverses of linear operators, SIAM J. Appl. Math., 29 (1975), pp. 103-109.

[64] S. L. CAMPBell AND C. D. MEYER, JR., Generalized Inverses of Linear Transformations, Pitman, London, 1979.

[65] P. ROBERT, On the group-inverse of a linear transformation, J. Math. Anal. Appl., 22 (1968), pp. 658-669.

[66] A. Albert, Conditions for positive and nonnegative definiteness in terms of pseudo inverse, SIAM J. Appl. Math., 17 (1969), pp. 434-440.

[67] E. KREINDLER AND A. JAMESON, Conditions for nonnegativeness of partitioned matrices, IEEE Trans. Automat. Control, AC-17 (1972), pp. 147-148.

[68] C. N. NETt, C. A. JACOBSON AND M. J. BALAS, Fractional representation theory: Robustness results with applications to finite dimensional control of a class of linear distributed systems, IEEE Conference on Decision and Control, San Antonio, TX, Dec. 1983.

[69] R. F. CURTAIN, Finite-dimensional compensators for parabolic distributed systems with unbounded control and observation, this Journal, 22 (1984), pp. 255-276.

[70] W. S. Levine AND M. ATHANS, On the determination of the optimal constant output feedback gains for linear multivariable systems, IEEE Trans. Automat. Control, AC-15 (1970), pp. 44-48.

[71] M. J. BALAS, The structure of discrete-time finite-dimensional control of distributed parameter systems, Proc. IEEE International Large Scale Systems Symposium, Virginia Beach, VA, 1982.

[72] M. S. Ghavsi AND J. J. Kelly, Introduction to Distributed-Parameter Networks with Application to Integrated Circuits, Holt, Rinehart and Winston, New York, 1968.

[73] D. S. BERNSTEIN, L. D. DAVIS AND D. C. HYLAND, The optimal projection equations for reduced-order, discrete-time modelling, extimation and control, submitted for publication. 